\title{
Archéologie préhistorique de la partie centrale du Main Ethiopian Rift (bassin lacustre de Ziway-Shala) : contribution à l'établissement de la séquence Late Stone Age d'Afrique orientale
}

\author{
François Bon 1 , Asamerew Dessie'2, Laurent Bruxelles ${ }^{3}$, \\ Axel Daussy ${ }^{4}$, Katja Douze ${ }^{5}$, François-Xavier Fauvelle-Aymar6, \\ Lamya Khalidi', Joséphine Lesur', Clément Ménard'9, \\ Ofer Marder ${ }^{10}$, Romain Mensan ${ }^{11}$, Guillaume Saint-Sever ${ }^{12}$
}

${ }^{1}$ UMR 5608 TRACES, Maison de la recherche, Université de Toulouse - le Mirail, 5 allées A. Machado, 31058 Toulouse Cedex 9, France; Honorary Research Fellow, School of Geography, Archaeology and Environmental Studies, University of the Witwatersrand, Johannesburg, South Africa.

2 Authority for Research and Conservation of Cultural Heritage, Addis Ababa, Ethiopia.

3 UMR 5608 TRACES, Maison de la recherche, Université de Toulouse - le Mirail, 5 allées A. Machado, 31058 Toulouse Cedex 9, France; Honorary Research Fellow, School of Geography, Archaeology and Environmental Studies, University of the Witwatersrand, Johannesburg, South Africa ; INRAP Méditerranée, 561 rue Étienne Lenoir, Km Delta, 30900 Nîmes, France.

${ }_{5}^{4}$ INRAP Grand Sud-Ouest, 13 Rue du Négoce, 31650 Saint-Orens-de-Gameville, France.

${ }^{5}$ UMR 5199 PACEA, Université de Bordeaux 1, Bât. B8, avenue des Facultés, 33405 Talence Cedex, France ; Evolutionary Studies Institute, University of the Witwatersrand, Johannesburg, South Africa.

${ }^{6}$ UMR 5608 TRACES, Maison de la recherche, Université de Toulouse - le Mirail, 5 allées A. Machado, 31058 Toulouse Cedex 9, France; Honorary Research Fellow, School of Geography, Archaeology and Environmental Studies, University of the Witwatersrand, Johannesburg, South Africa.

7 UMR 5133 Archéorient, Maison de l'Orient et de la Méditerranée, 7 rue Raulin, 69007 Lyon, France.

${ }^{8}$ UMR 7209 Archéozoologie, Archéobotanique : Sociétés, Pratiques et Environnements, CP 56, 55 rue Buffon, Muséum National d'Histoire Naturelle, 75005 Paris, France.

9 UMR 5608 TRACES, Maison de la recherche, Université de Toulouse - le Mirail, 5 allées A. Machado, 31058 Toulouse Cedex 9, France.

${ }^{10}$ Faculty of Humanities and Social Sciences, Department of Bible, Archaeology and Ancient Near East, Ben-Gurion University of the Negev, Beersheva, Israël.

${ }^{11}$ UMR 5608 TRACES, Maison de la recherche, Université de Toulouse - le Mirail, 5 allées

A. Machado, 31058 Toulouse Cedex 9, France.

12 UMR 5608 TRACES, Maison de la recherche, Université de Toulouse - le Mirail, 5 allées A. Machado, 31058 Toulouse Cedex 9, France. 


\section{Problématique d'étude et état de l'art}

Débutée en 2007, cette mission a eu pour objectif de contribuer à l'enrichissement de nos connaissances sur le Late Stone Age (LSA) de la Corne de l'Afrique grâce à la constitution de nouveaux référentiels archéologiques. Le choix de la région d'étude, celle du bassin lacustre de Ziway-Shala, située au cœur de la vallée du Rift, a été motivé par plusieurs raisons (Bon et al., 2006). En premier lieu, il nous a semblé pertinent de nous interroger sur le rôle éventuel de cet environnement lacustre au regard des mutations économiques reconnues en d'autres endroits d'Afrique de l'Est au cours du Late Stone Age, qu'il s'agisse du développement d'une économie halieutique au début de l'Holocène, selon le modèle proposé par Sutton (1977), ou bien du développement ultérieur d'économies pastorales tel qu'il a pu notamment être décrit au Kenya ou à Djibouti (Lane, 2004 ; Gutherz, 2008). En second lieu, le choix de cette région des lacs a également été dicté par les documents archéologiques d'ores et déjà disponibles. Si les plus célèbres demeurent les stations Middle Stone Age de Gademotta et Kulkuletti, localisées à quelques encablures du lacs Ziway (Wendorf et Schild, 1974 ; Douze, 2012 ; Sahle et al., sous presse), notre attention s'est davantage portée sur les sites plus récents décrits par Humphreys à Macho Hill et Waso Hill (Humphreys, 1978) ainsi que sur les référentiels réunis à Aga Dima («Red Stone Hill ») par Gallagher (1977a), la présence d'industries LSA ayant été également signalée dans les coupes naturelles de la rivière Bulbula lors des prospections géologiques conduites par Street (Gasse et Street, 1978; Street, 1979 ; Gasse et al., 1980 ; Brandt, 1986). Autre point de comparaison utile, c'est également dans cette région qu'ont été décrites certaines industries sub-contemporaines, en particulier sur le site de Dalacha, au pied du volcan Alutu (Gallagher, 1972), comme auprès d'artisans tanneurs utilisant couramment, il y a encore quelques décennies, des grattoirs à peaux armés de tranchants d'obsidienne (Gallagher, 1974 et 1977b). Enfin, il s'avère que cette région des lacs du Main Ethiopian Rift (MER) a bénéficié de plusieurs programmes permettant de disposer d'une couverture géologique et environnementale, en particulier de nombreux référentiels pédo-sédimentaires (Grove et al., 1975 ; Laury et Albritton, 1975 ; Gasse et Street, 1978 ; Street, 1979 ; Gasse et al., 1980 ; Gillespie et al., 1983 ; Le Turdu et al., 1999 ; Benvenuti et al., 2002 ; Umer et al., 2004 ; Sagri et al., 2008 ; Carnicelli et al., 2009).

\section{Déroulement de la mission}

Au cours des deux premières campagnes (2007-2008), nous nous sommes basés sur les données préalablement existantes et avons poursuivi l'exploration et la comparaison de plusieurs contextes topographiques qui avaient chacun livré des témoignages plus ou moins abondants d'occupation rapportés au $L S A$ : nous sommes donc retournés sur les collines de Macho et Waso (cf. travaux de Humphreys; Bon et al., 2006 et 
Annexe 4), dans les abris sous roche d'Aga Dima (cf. travaux de Gallagher et Annexe 3) ainsi que sur les bords de la rivière Bulbula (cf. travaux de Street; Fig. 1). Si chacun de ces contextes apporte un éclairage complémentaire sur la diversité des modes d'installation humaine dans cette zone - diversité qu'il est potentiellement intéressant de mettre en relation avec l'évolution du paysage au cours du temps, en vertu notamment des variations du niveau des lacs (collines et rochers surplombant la plaine lors des phases de transgression, occupations au plus près de la rivière ne pouvant intervenir que lors des épisodes de régression) -, il s'avère que seul le contexte pédo-sédimentaire offert par les berges de la rivière Bulbula a été susceptible de répondre à nos attentes. En effet, les traces d'occupations LSA observées sur les collines de Macho et de Waso sont contenues dans des formations pédo-sédimentaires (paléosols polycycliques) dont il demeure très difficile d'apprécier le calage chronologique, en l'absence notamment de toute conservation de vestiges organiques datables (Bon et al., 2006), tandis que les abris d'Aga Dima ne possèdent guère de remplissages permettant d'établir une séquence stratigraphique précise (cf. Annexe 3).

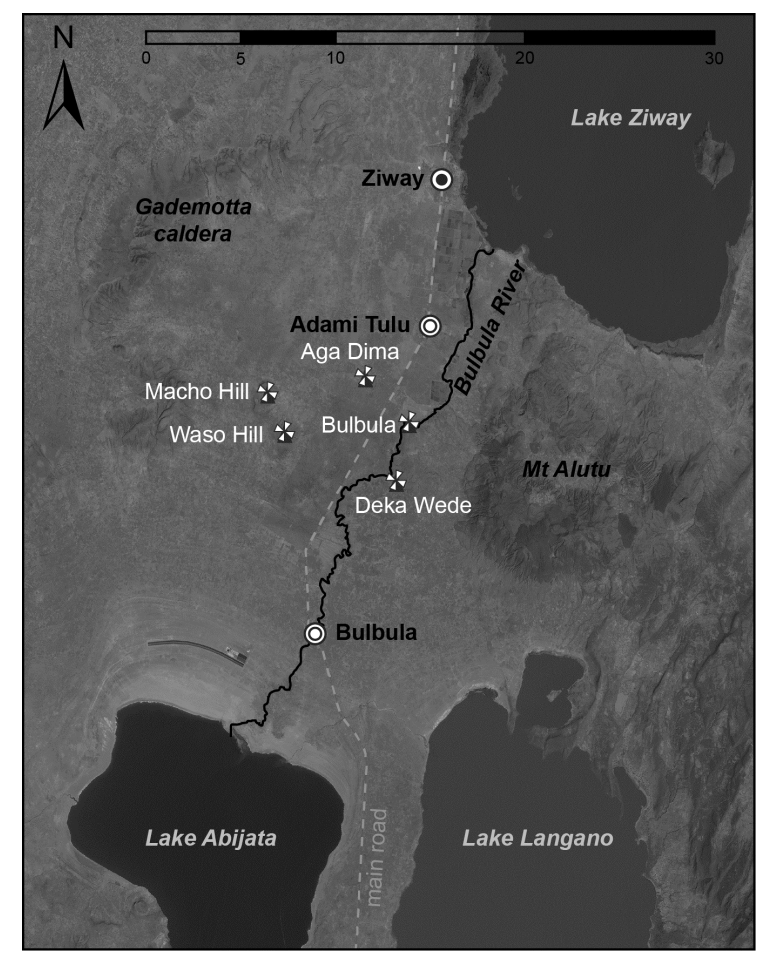

Fig. 1 - Image satellite de la zone étudiée par le projet et principaux sites LSA (moulins). Dans un souci de lisibilité, le cours de la rivière Bulbula a été redessiné (image Aster, échelle en kilomètres) (DA0 : C. Ménard). 
Ainsi, dès 2008, compte tenu de la qualité des enregistrements pédosédimentaires qu'elles possèdent, notre attention s'est principalement concentrée sur les rives de la Bulbula. Mieux que les deux précédents, ce contexte offre en effet l'opportunité de replacer les occupations humaines au sein de l'évolution du paysage dans la longue durée, en permettant notamment de les corréler avec les variations des niveaux des lacs. Par ailleurs, a contrario des paléosols présents sur les collines avoisinantes, plusieurs des formations pédo-sédimentaires observées le long des rives de la Bulbula conservent des vestiges organiques (faune, charbon, coquillage) permettant ainsi, outre la possibilité d'obtenir des datations, d'aborder certains pans de l'économie des groupes concernés.

C'est donc à ce contexte, celui des rives de la Bulbula et de ses affluents, que les trois campagnes de terrain suivantes (2009-2011) furent principalement dédiées, poursuivant la fouille et la description du secteur baptisé Bulbula 1 (Aneno Melka Gademotta) découvert en 2008 et comparant ce dernier aux données géologiques et archéologiques de plusieurs autres secteurs, en particulier ceux d'Aneno, du Deka Wede et de Kurkura (cf. Fig. 2). Grâce à la confrontation des données recueillies dans ces différents secteurs, sous la forme de levées de coupes (Laurent Bruxelles et Romain Mensan) et de nombreux sondages à vocation archéologique et géologique (dirigés par Asamerew Dessie, François Bon, Katja Douze et Clément Ménard) ainsi qu'un enregistrement topographique du secteur de Bulbula 1 (Axel Daussy), nous sommes ainsi parvenus à restituer une séquence géologique détaillée de cette zone au cours des derniers 40 milliers d'années et à apporter des éclairages substantiels sur l'occupation humaine de cette région au cours de plusieurs phases appartenant au MSA final et au LSA.

À cela, il faut ajouter que, dès 2007, nous avons entrepris des prospections géologiques dans le but de localiser et de décrire les sources d'obsidienne les plus proches, susceptibles d'avoir été employées par les groupes humains concernés par nos recherches. Cette étude a pris une dimension nouvelle depuis 2010 grâce à l'intervention de Lamya Khalidi, qui a conduit un programme de prospection, d'échantillonnage et d'analyses approfondi, en collaboration avec Bernard Gratuze (UMR 5060 IRAMAT). Trois missions de terrain conduites entre 2010 et 2012 ont permis d'échantillonner un grand nombre de coulées d'obsidienne provenant du volcan Alutu et de la Gademotta Ridge (Kulkuletti, Gademotta, Andola Chabbi), ainsi que d'autres secteurs composant la région du bassin de Ziway-Shala. 


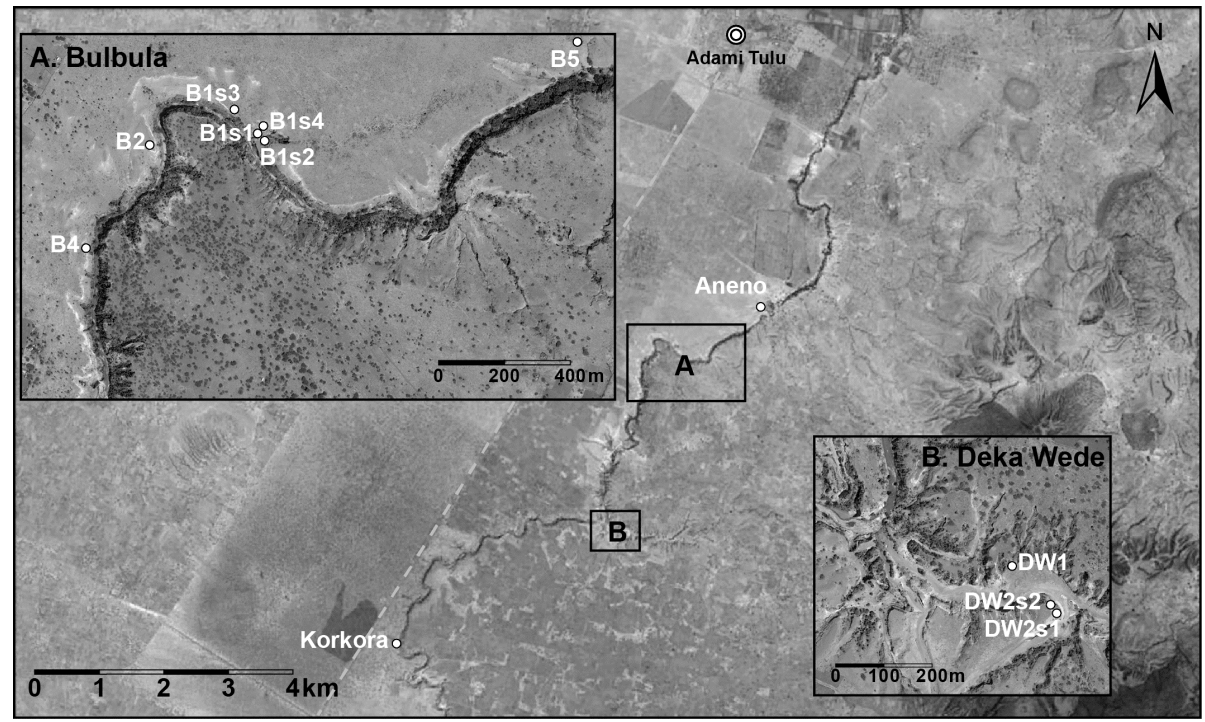

Fig. 2 - Image satellite de la partie centrale de la plaine de la Bulbula et vues rapprochées des principales zones étudiées (Bulbula et Deka Wede). Images Google Earth (DA0 : C. Ménard).

Enfin, ajoutons que notre mission a apporté un support logistique à l'étude ethnoarchéologique des productions céramiques contemporaines régionales, suivant un programme coordonné par Jessie Cauliez (UMR 5608 TRACES). Nous avions en effet constaté que si la question des traditions lithiques sub-actuelles a déjà fait l'objet de plusieurs recherches (cf. en particulier les travaux précédemment cités de Gallagher), tel n'était pas le cas à l'égard des traditions céramiques, limitant d'autant l'interprétation de cette catégorie de vestiges lorsqu'on les rencontre en contexte archéologique.

\section{Contexte géologique et archéologique de la plaine de la Bulbula}

La plaine de la Bulbula relie les lacs Ziway et Abijata, distants I'un de l'autre, selon un axe nord-est/sud-ouest, d'environ $28 \mathrm{~km}$. À l'est, cette plaine est bordée par la caldera du volcan Alutu tandis qu'à l'ouest, elle est cernée par les collines de Waso et de Macho, qui constituent l'extrémité méridionale du Gademotta Ridge. Entre ces reliefs, son étendue s'établit sur environ $10 \mathrm{~km}$ de large. Sur la plus grande partie de son tracé, l'altitude de cette plaine est comprise entre 1640 et 1700 mètres, formant un assez vaste replat dominé, d'une part, par les hauteurs de Waso (env. 1780 mètres) et celles, beaucoup plus élevées, de l'Alutu (2 328 mètres) ; elle surplombe, d'autre part, les niveaux des lacs Ziway (1 636 mètres) et Abijata (1 578 mètres). 
Cette plaine, aujourd'hui occupée par une savane arborée abritant de nombreux villages, est partagée par la profonde incision pratiquée par la Bulbula. Cette rivière est le déversoir du lac Ziway, dont elle dirige les eaux vers celles du lac Abijata. Tout au long du tracé de la Bulbula, ces eaux permanentes sont renforcées par celles, intermittentes, de nombreux tributaires. C'est particulièrement le cas le long de sa rive gauche car celle-ci, longeant les contreforts du volcan Alutu, collecte saisonnièrement les eaux de plusieurs affluents, dont l'un des plus importants est le Deka Wede.

Tout au long de son tracé, la Bulbula incise, souvent sur plusieurs dizaines de mètres, la plaine du même nom et c'est ainsi que, sur la majeure partie de son cours, ses berges se présentent sous la forme d'escarpements verticaux pouvant atteindre 30 à 50 mètres de dénivelé. Et, en définitive, les points où l'on peut aisément atteindre le cours d'eau sont assez peu nombreux: outre quelques sentiers escarpés ménagés à flanc de versant pour permettre le passage des troupeaux, il s'agit principalement de secteurs où des tributaires saisonniers ont entaillé ses berges, créant ainsi des dépressions facilitant l'accès à celles-ci, à l'image de Bulbula 1 (Fig. 3).

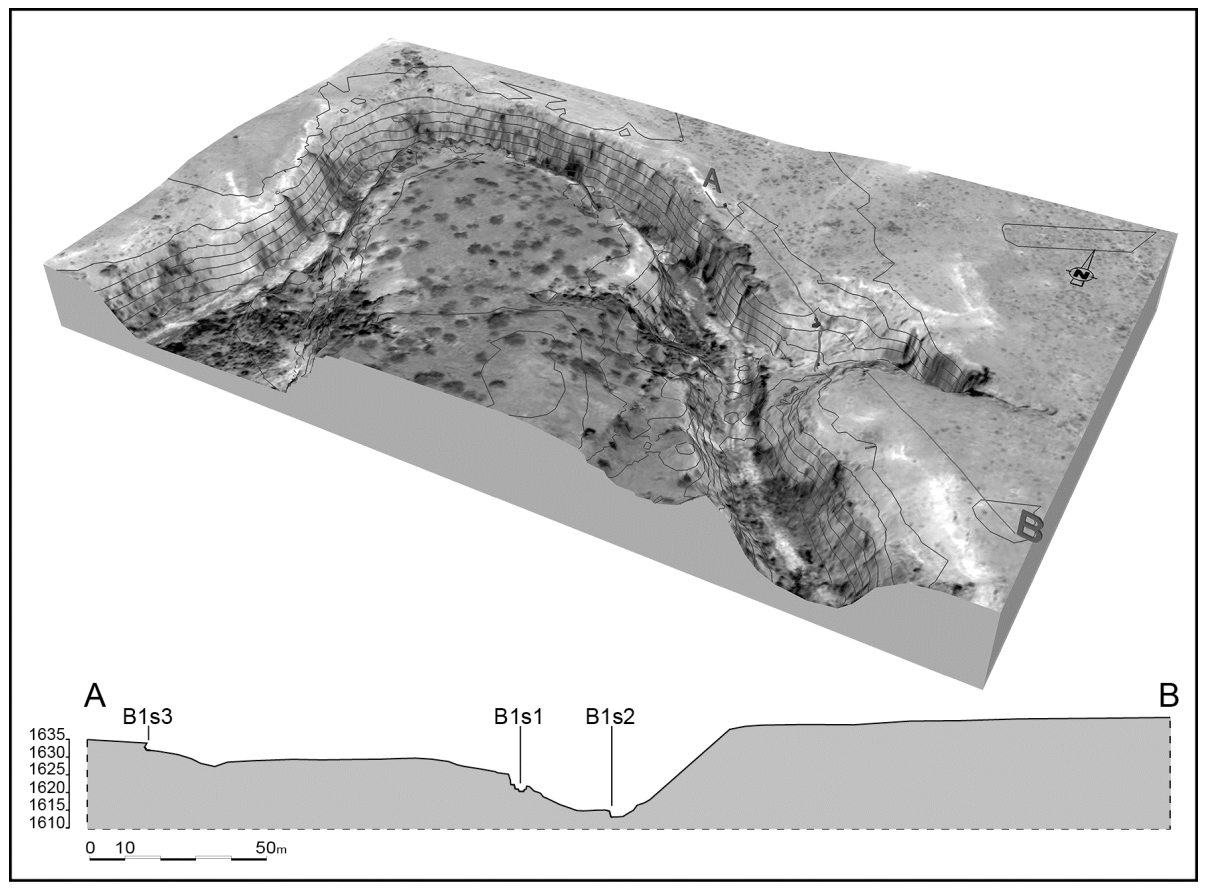

Fig. 3 - Vue isométrique de la zone Bulbula 1 (courbes de niveaux générées à partir du relevé topographique; image Google Earth). Profil indiquant la position des sites archéologiques appartenant à des phases d'occupations distinctes, altitudes en mètres au dessus du niveau de la mer (DA0 : A. Daussy). 
Les deux principaux secteurs où nous sommes intervenus ont été baptisés Bulbula (B1 à B5) et Deka Wede (DW1 et 2). Bulbula 1 correspond au débouché d'un thalweg empruntant un ancien cours de la Bulbula et rejoignant à cet endroit son tracé actuel. C'est là que, dès 2008, ont été réunis de premiers éléments de description de la séquence géologique correspondant au remplissage de cette plaine fluvio-lacustre tandis que nous entreprenions l'exploration de plusieurs sites (B1s1, B1s2, B1s3 et B1s4). Au cours des campagnes 2008 et 2009, nous avions toutefois d'ores et déjà commencé à étendre nos prospections vers le sud $(B 2, B 4)$ et vers le nord (B5). En 2010 et 2011, nous avons amplifié ces recherches le long de la Bulbula en multipliant les observations sur les secteurs d'Aneno (au nord) et du Deka Wede et de Kurkura (au sud). Dans chacun d'entre eux, nous avons procédé au relevé de la séquence géologique, à l'échantillonnage systématique des formations, ainsi qu'à des prélèvements en vue de datations par différentes méthodes (en particulier sur les coquillages et les pierres ponces). Des échantillons sédimentaires ont également été prélevés afin de conduire une analyse palynologique (tests effectués par Didier Galop, UMR 5602 GEODE).

Parallèlement, comme nous venons de l'indiquer, des fouilles ont été pratiquées en plusieurs endroits (10 secteurs en tout). Quatre secteurs de Bulbula 1 (secteurs 1 à 4) ont permis la réunion de séries archéologiques significatives, la collecte d'échantillons plus limités en vue de comparaison ayant été réalisée à Bulbula 2, Bulbula 4 et Bulbula 5. L'autre zone où nous sommes intervenus archéologiquement est celle du Deka Wede, où trois secteurs ont été explorés (DW1 et DW2 secteurs 1 et 2 ; Fig. 4). À cela, s'ajoute le fruit de prospections réalisées dans la plaine à quelque distance des rives de la Bulbula, en particulier dans les environs de Bulbula 1. L'analyse de ces différents assemblages archéologiques ainsi que leur comparaison avec les informations recueillies auparavant dans la même zone par Gallagher (1977a), Humpreys (1978) et Street (1979), apportent de nouveaux éclairages afin de décrire la nature et l'évolution des industries lithiques de cette région dans la longue durée. Cela concerne à la fois l'identité technologique du MSA final, le développement des industries microlithiques lors des phases finales du Pléistocène et du début de I'Holocène (Ménard et al., soumis ; Ménard, thèse en cours; Behailu Habte, 2013), ou bien encore la perduration de traditions lithiques lors des périodes historiques. Certains de ces contextes ont par ailleurs livré des vestiges fauniques permettant d'établir une corrélation entre cette évolution industrielle et celle des stratégies d'acquisition des ressources alimentaires (Lesur, étude en cours). La conjugaison de ces différentes études et analyses permet de proposer une première synthèse de l'évolution du paysage de la plaine de la Bulbula et de sa fréquentation par l'Homme au cours des derniers 40000 ans. 


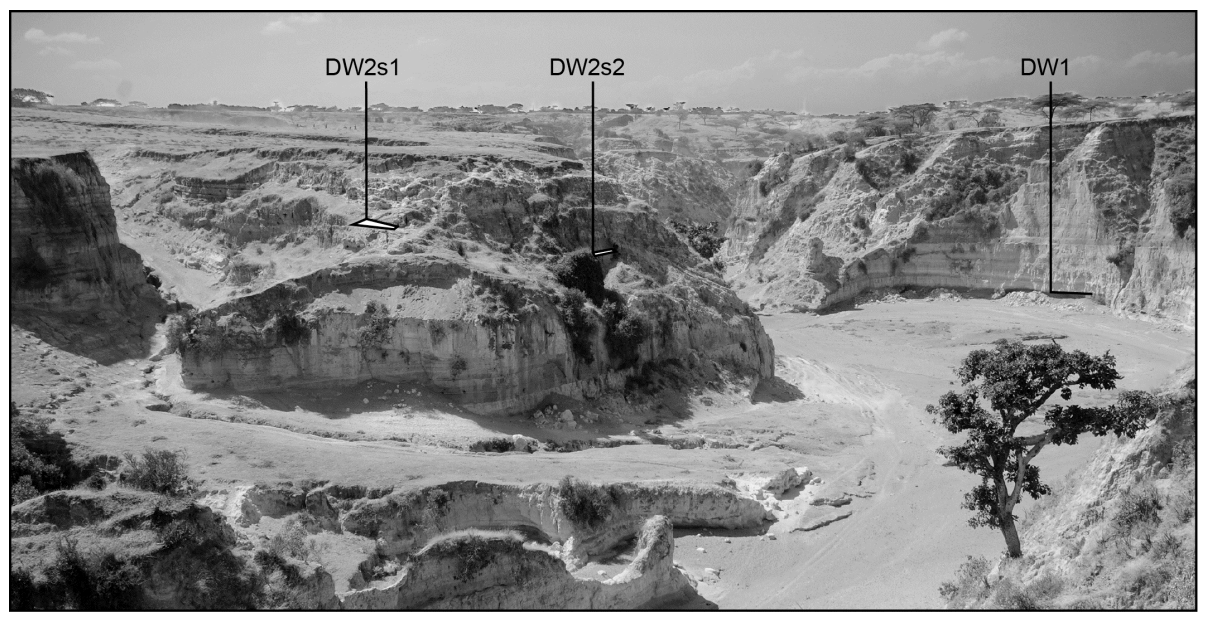

Fig. 4 - Photographie orientée vers le sud-ouest de la zone du Deka Wede avec emplacement des sites fouillés (DA0 : C. Ménard).

\section{Synthèse : histoire du paysage et dynamique d'occupation humaine dans la plaine de la Bulbula}

Le premier événement géologique enregistré correspond à une intense activité volcanique, laquelle se traduit par de puissants dépôts volcanoclastiques qui servent de substrat au lit actuel de la Bulbula en plusieurs endroits (Bulbula 1 et Deka Wede). Aucun élément ne permet aujourd'hui d'attribuer un âge à cette formation, que l'on peut simplement mettre en relation avec une activité ancienne du volcan Alutu. D'après les données disponibles à ce sujet, la formation de la caldera de l'Alutu semble remonter à environ $240000 \mathrm{BP}$, une forte activité étant ensuite enregistrée vers 150000 BP (d'après Le Turdu et al., 1999). En tout état de cause, ces dates offrent une fourchette crédible afin d'estimer l'âge approximatif de ces dépôts volcano-clastiques (circa 250-150 000 BP).

C'est ensuite qu'une première séquence lacustre se met en place, laquelle se traduit d'abord par d'épais dépôts alluviaux puis par des limons et s'achève par une formation gréseuse indurée (désignée « dalle » et qui constitue un repère stratigraphique important dans le paysage). Aucun élément ne permet actuellement d'attribuer un âge à cette séquence, qui correspond au plus haut niveau de lac enregistré dans le paysage («Megalake », correspondant vraisemblablement au «synthem 1 » décrit par Benvenuti et ses collaborateurs; Benvenuti et al., 2002). D'après les interprétations fournies par des études géologiques antérieures à la nôtre, il est vraisemblable que le plus haut niveau de lac corresponde à l'OIS5e, soit aux alentours de $120000 \mathrm{BP}$, et que cet épisode s'achève peu ou prou 
avec I'OIS5a, soit vers $80000 \mathrm{BP}$. Aucun vestige archéologique n'a été observé dans l'ensemble de ces formations ${ }^{13}$.

Postérieurement, à une date toujours inconnue pour laquelle nous pouvons simplement proposer à titre d'hypothèse qu'elle coïncide avec I'OIS4, soit aux alentours de 70000 BP, la régression du lac entraîne un épisode d'érosion intense, qui ne laisse subsister en surface de la plaine que cette «dalle » évoquée précédemment. Cette dernière joue alors le rôle de surface d'érosion sur l'ensemble de la plaine, contraignant par sa résistance le développement de la végétation et limitant ainsi la formation de sols. Sans doute peut-on imaginer que, durant plusieurs milliers d'années, la plaine de la Bulbula s'est donc présentée comme une étendue inhospitalière, alternant remontées des eaux et périodes d'assèchement et d'érosion mettant à nu cette « dalle ».

\section{Les premières traces d'occupation humaine enregistrées dans la plaine de la Bulbula : les derniers temps du Middle Stone Age}

Plus tard, lors d'une longue période de basses eaux, un sol parvient toutefois à se former progressivement, grâce à l'apport de matériaux volcaniques remobilisés par colluvionnement, sur lesquels se développe une végétation entraînant la formation d'un sol. Nous ne savons pas quand débute la formation de ce sol, mais possédons quelques indications sur la fin de son fonctionnement: il est recouvert par une formation de ponces correspondant à l'Abernosa pumice member attribuée par Street à une période immédiatement postérieure à $22000 \mathrm{BP}$ (Street, 1979). Quoi qu'il en soit, ce sol est conservé en de nombreux points de la plaine actuelle de la Bulbula et contient en plusieurs endroits des indices d'occupation humaine (B1s3, B4; Fig. 5). Nous pouvons par ailleurs le mettre en relation avec des formations alluviales contemporaines observées dans le Deka Wede (DW1).

Ces différents contextes détiennent, comme nous l'évoquions, des traces d'occupations humaines. Au Deka Wede, c'est à ce paléosol que se rapporte une industrie datée par Street des environs de 27000 BP et qualifiée par elle, sur la foi d'une observation menée par Clark, Brandt et Kurashina de "Early Late Stone Age », grâce à sa composante laminaire (conclusions reproduites notamment dans Brandt, 1986). Cette date fournie par Street correspond relativement bien à celle que nous sommes parvenus à obtenir sur le même niveau : $29090 \pm 160$ BP (DW1). En revanche, la comparaison de plusieurs de ces assemblages (B1s3, B4 et DW1, dont seul le dernier a pu être daté) permet de corriger l'attribution de cette industrie à du ELSA : si ces séries comportent en effet une production laminaire bien exprimée, celle-ci s'inscrit dans la tradition de

\footnotetext{
${ }^{13}$ La datation de ce très haut niveau de lac («Megalake ») est l'objet de plusieurs interprétations, dont certaines tendraient plutôt à l'attribuer à l'OIS7 (cf. hypothèse émise par Laury et Albritton dans leur interprétation de la séquence de Gademotta ; Laury et Albritton, 1975).
} 
savoir-faire évoquant les technologies du MSA, comme le suggère notamment l'usage omniprésent de la méthode Levallois (Ménard et al., soumis). La technologie dominante représentée dans ces trois ensembles suit en effet une méthode Levallois récurrente bipolaire, dont les produits laminaires qui en sont issus sont soit transformés par retouches convergentes et amincissement de la base, soit utilisés bruts ; en ce cas, il s'agit souvent de couteaux à dos de débitage. L'ensemble de ces caractères désigne ces industries comme profondément ancrées dans une tradition technologique héritée du MSA, dont elles incarnent un stade final.

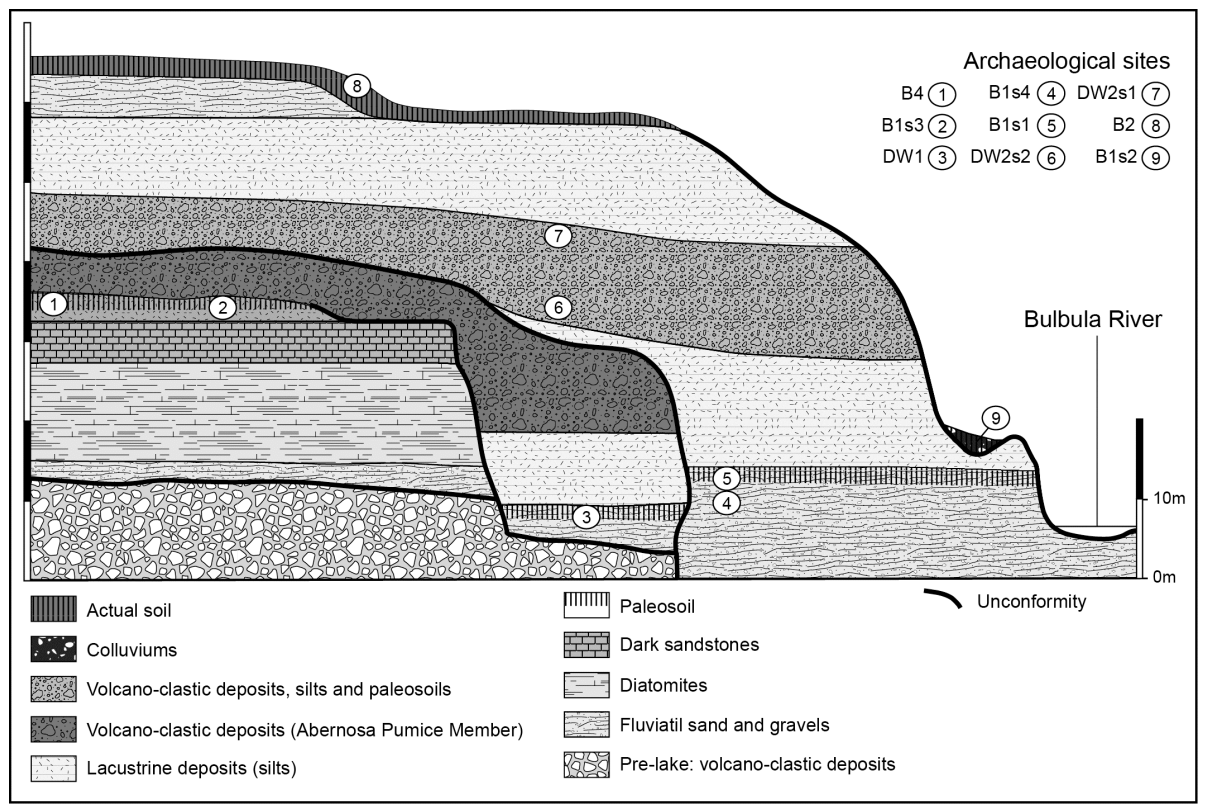

Fig. 5 - Log stratigraphique des formations géologiques reconnues dans la plaine de la Bulbula. Les sites archéologiques sont représentés à titre indicatif (le contexte et l'emplacement réels de B1s4 et de B1s1 ne peuvent pas être restitués sur cette vue) (DA0 : L. Bruxelles).

Malheureusement, dans la plupart des secteurs où nous l'avons observé, ce sol n'a pas conservé de vestiges fauniques et seule la coupe de DW1 livre quelques fragments d'os, associés à des charbons (dont un échantillon a livré la date évoquée plus haut). Toutefois, le secteur de B1s3 a livré un vestige osseux : un fragment proximal d'humérus d'hippopotame (détermination: Jean-Renaud Boisserie), dont il s'avère que la surface articulaire porte des stigmates compatibles avec un emploi comme percuteur, hypothèse qui méritera d'être confirmée par des analyses plus approfondies. 


\section{Développement du Late Stone Age à la charnière du Pléistocène et de l'Holocène}

Postérieurement à l'épisode de dépôt de ponces évoqué précédemment (Abernosa pumice member), soit sans doute aux environs de 22 à 18000 $\mathrm{BP}$ (c'est-à-dire en plein OIS2), un nouvel épisode d'érosion est attesté. Les nombreuses dépressions creusées sur les flancs du canyon de la Bulbula lors de cet épisode se comblent ensuite à la faveur de différents phénomènes sédimentaires. Dans le Deka Wede, un épais dépôt de limons traduit l'envahissement ponctuel du canyon de la Bulbula par les eaux du lac Abijata. Puis, au Deka Wede comme à Bulbula 1, on assiste à l'apport de colluvions issues de la plaine sus-jacente, lesquelles se mêlent aux alluvions de la Bulbula. Dans les thalwegs hérités de l'épisode d'érosion inaugurant cette nouvelle phase, l'humidité favorise la formation d'un couvert végétal plus dense, lequel se traduit par la formation de sols mieux marqués ici que dans la plaine, plus aride.

Le plus ancien contexte est celui de B1s4 (12 040-11 $\left.840 \mathrm{cal} \mathrm{BC}^{14}\right)$, qui précède de quelques siècles les occupations de B1s1, site multi-stratifié pour lequel nous disposons de deux dates quasiment identiques entre le sommet et la base de la séquence : 11 471-11 287 et $11460-11300 \mathrm{cal}$ BC (Fig. 6). Ici, outre la protection d'un vallon surplombant les gorges de la Bulbula, les groupes humains de la fin du Pléistocène ont bénéficié de la protection naturelle d'un petit abri formé par l'érosion de la «dalle» évoquée précédemment. L'occupation humaine semble ensuite de nouveau s'interrompre lors de l'épisode du Younger Dryas, pour ne reprendre que vers $9800 \mathrm{cal} B C$ à DW2s2 $(9820-9360 \mathrm{cal} \mathrm{BC})$ puis à DW2s1 (9350-9240 cal BC), lors des premiers siècles de l'Holocène (Fig. 6).

Les industries de ces différents sites correspondent à un Late Stone Age dénué cette fois-ci de tous caractères hérités du MSA, où coexistent des lames sur lesquelles sont aménagés des instruments de type grattoirs, burins, couteaux à dos, et de petites lames et lamelles fournissant des pointes à dos (B1s1). Toutefois, chacune de ces industries possède des caractères propres, illustrant une variabilité inédite des traditions techniques rangées sous la bannière du LSA. Ces différences s'expriment notamment dans la famille des pièces à dos et des armatures, lesquelles conditionnent l'existence de schémas opératoires de production contrastés (Ménard et al. soumis; Ménard, thèse en cours). Ainsi par exemple, l'industrie la plus récente, celle de DW2s1, se singularise par son degré de microlithisme: la production laminaire tend à disparaître tandis que l'intention lamellaire devient omniprésente et fournit des pointes microlithiques d'un type inédit.

14 Datations calibrées obtenues à partir de la courbe Intcal 09 (Reimer et al., 2009); intervalles exprimés à 2 sigmas. 


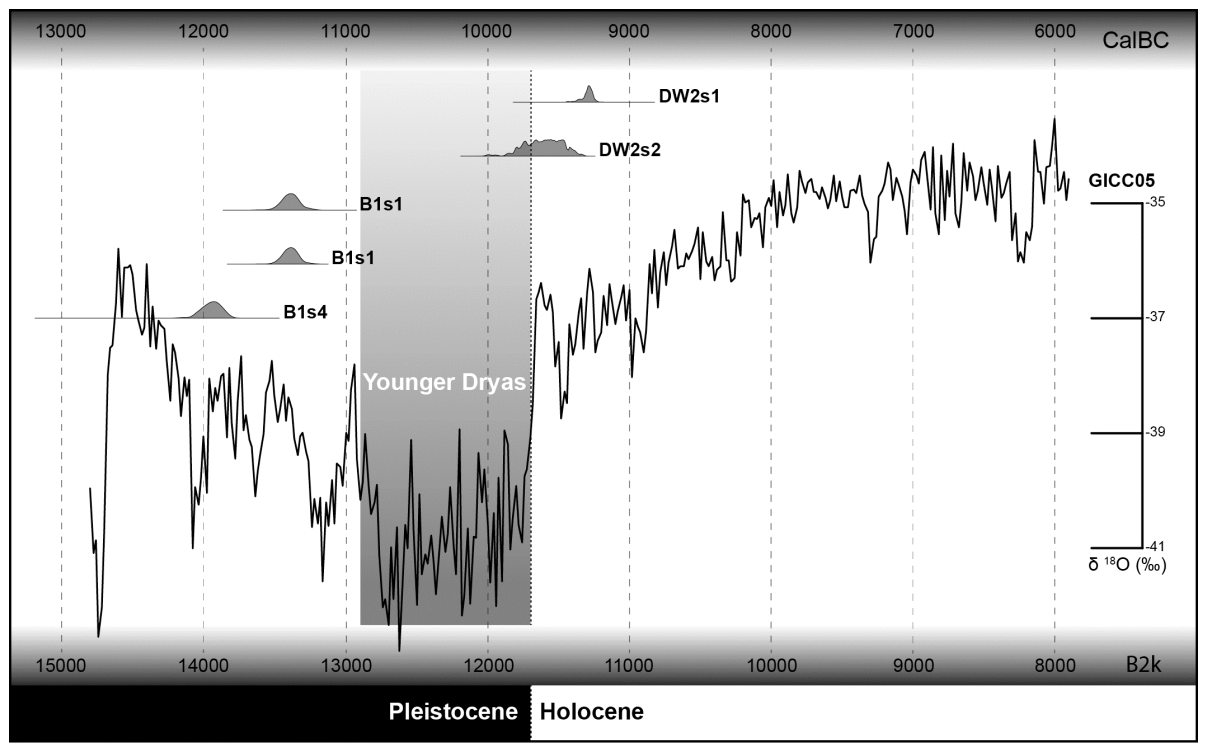

Fig. 6 - Datations radiocarbones des sites archéologiques de la fin du Pléistocène et du début de I'Holocène. Pour comparaison, courbe GICC05. Datations calibrées avec la courbe IntCal09 (Reimer et al., 2009); données GICC05, NGRIP dating group (Rasmussen et al., 2006) (DA0 : C. Ménard).

Il convient surtout de souligner le fait que c'est lors de ce dernier épisode que l'on constate, dans un environnement désormais davantage marqué par l'influence lacustre, un changement économique majeur: tandis que les faunes associées aux premières occupations (B1s1, DW2s2) étaient largement dominées par les mammifères terrestres, les poissons occupent ensuite une part dominante (DW2s1). Ces occupations, datées de la seconde moitié du $X^{\mathrm{e}}$ millénaire cal $\mathrm{BC}$, traduisent donc un changement techno-économique significatif. Les lignes qui suivent reproduisent les premières conclusions de l'analyse en cours entreprise par Joséphine Lesur. Précisons tout d'abord que les riches corpus livrés par B1s1 et DW2s2 sont marqués par une très forte fragmentation des os, limitant d'autant leur attribution à un taxon. Ainsi, à B1s1, plus de 19000 restes ont été dénombrés mais seuls 1860 ont pu être déterminés; à DW2s2, cette proportion tombe à 121 pour $4964,97 \%$ des restes étant composés d'esquilles dont la très grande majorité fait moins d'un centimètre de longueur, attestant d'une très grande fragmentation. Celle-ci semble en grande partie résulter de la fracturation volontaire des os par les occupants du site pour la consommation de la moelle, mais aussi d'une importante carbonisation dont les traces sont visibles sur plus d'un tiers des restes. Quoiqu'il en soit, dans les deux cas, le spectre faunique est largement dominé par la capture de mammifères terrestres, la majorité provenant de bovidés de grande ou moyenne taille. À cela, s'ajoutent des fragments d'œuf d'autruche, dont certains présentent des traces de 
façonnage pour la réalisation de perle à B1s1 (6 exemplaires, entiers ou fragmentés). Par la suite, tandis que l'équipement lithique se transforme radicalement en faveur d'une forte microlithisation des armatures comme de l'ensemble des instruments, on assiste donc à un très profond changement de diète alimentaire : si le secteur 1 de Deka Wede 2 (DW2s1) n'a livré que quelques centaines de restes, près de $75 \%$ proviennent de poissons, notamment Barbus sp. et Labeo sp.

Ainsi, plusieurs de ces sites présentent des profils très différents quant à l'exploitation animale. À B1s1 comme à DW2s2, les occupants semblent avoir principalement chassé des bovidés sauvages comme le bubale ou la gazelle; quelques siècles plus tard, à DW2s1, l'exploitation de la faune s'est concentrée presque exclusivement sur la pêche, très probablement dans les lacs situés à proximité. Dans le premier cas, les différents taxons déterminés spécifiquement, à savoir le bubale, la gazelle de Grant et le lièvre, attestent tous d'un paysage de savane herbeuse ou broussailleuse qui peut être sèche mais avec des points d'eau toujours à proximité. Dans le second, la suite des analyses doit permettre d'affiner les résultats, notamment en ce qui concerne les lieux et saisons de capture des poissons, afin de mieux interpréter la portée d'un tel changement économique en faveur de la pêche.

\section{À la recherche du Néolithique}

Postérieurement à ces occupations et, d'un point de vue géologique, dans la continuité parfaite avec ce qui a été décrit précédemment, on assiste alors à une remontée plus importante du niveau des lacs qui conduit à leur réunion («Macrolake»; synthem 3 de Benvenuti et al., 2002). Cette phase se traduit par d'importants dépôts de limons, scandés dans leur partie supérieure par un horizon sableux contenant deux remarquables niveaux de coquillages datés des environs de $4900 \mathrm{cal} \mathrm{BC}(6000 \pm 35$ BP et 5985 $\pm 35 \mathrm{BP}$ ). Puis, les dernières expressions de cet environnement lacustre se matérialisent par la formation de Gilbert deltas (B5), dont nous ne connaissons pas la chronologie avec précision mais pouvons néanmoins proposer qu'ils soient relativement récents, à l'image de la seule date actuellement disponible pour cette formation sommitale : $3650-3520 \mathrm{cal}$ BC. II convient de préciser que, contrairement aux études antérieures qui concluaient en faveur d'un ou plusieurs courts épisodes de régression dans la première moitié de l'Holocène, nos propres observations tendent à démontrer l'existence d'une transgression continue du lac entre circa 9200 et $3500 \mathrm{cal} \mathrm{BC}$. En effet, si cette longue période est sans doute marquée par quelques oscillations, rien $n$ 'indique l'existence d'une régression entraînant la séparation des lacs au cours de cette première partie de l'Holocène.

C'est seulement en effet après 3500 cal BC que l'on assiste à une nouvelle baisse du niveau des eaux, conduisant à la séparation des lacs et à la mise en place progressive du paysage actuel. Comme à l'époque du 
paléosol de B1s3 et B4 (MSA final), un sol se développe alors largement dans la plaine, livrant fréquemment des indices plus ou moins concentrés d'occupations humaines récentes, dominées alors par le débitage d'éclats et la confection de grattoirs, en l'absence de toute production lamellaire (B2 et B5; Fig. 5). C'est également à cet épisode que correspond le paléosol supérieur relevé en $B 1 s 2$ et daté $d u \mathrm{VII}^{\mathrm{e}}-\mathrm{VIII} \mathrm{I}^{\mathrm{e}}$ siècle de notre ère (652-766 AD). Pour la première fois en séquence, la céramique fait son apparition ainsi que, sous toute réserve, les animaux domestiques, la présence d'un reste de bœuf (Bos taurus) demeurant cependant hypothétique. Ici comme dans les autres sites contemporains de cette phase, les productions laminaires et/ou lamellaires ont totalement disparu au profit de l'obtention d'éclats selon une méthode discoïde. L'outillage se borne principalement à des éclats retouchés et à un grattoir sur éclat (qui évoque le type gudit scraper décrit à Aksum; Phillipson, 2000), mais I'on observe aussi la présence d'une pièce à dos sous la forme d'un lunate, également confectionné sur éclat et non sur lamelle.

\section{Bilan et perspectives}

La séquence archéologique que nous sommes parvenus à appréhender dans la plaine de la Bulbula est par essence discontinue: les phases d'installation humaine y sont naturellement étroitement tributaires des cycles de régression lacustre, mais aussi d'autres facteurs tels qu'à l'inverse la mise place d'un milieu trop aride ou bien encore marqué par des retombées volcaniques ayant pu rendre, pour quelques temps au moins, les conditions de vie inhospitalières. Cette séquence est également le reflet, non seulement de conditions environnementales changeantes, mais aussi de la conservation de traces régulièrement soumises à une forte érosion du milieu qui les contient. Tous ces paramètres conjugués expliquent que notre connaissance de la Préhistoire locale ne porte en définitive que sur quelques fenêtres temporelles, dont le degré de résolution est d'ailleurs assez contrasté. II n'en demeure pas moins que chacune de ces fenêtres apporte des éclairages nouveaux sur la Préhistoire récente de la vallée du Rift, contribuant à enrichir le panorama dressé auparavant et à répondre à certaines des questions demeurées jusqu'alors en suspend et qui justifièrent le lancement de ce programme (Bon et al., 2006).

Parmi ces questions, figurait la réalité d'une phase précoce du LSA, d'après la découverte faite par Street d'une industrie à caractère laminaire datée des environs de 27000 BP dans une coupe du Deka Wede (Street, 1979, interprétation reprise par Brandt, 1986). En définitive, nos recherches dans ce secteur (DW1) comme l'exploration de plusieurs autres sites livrant un mobilier comparable (B1s3, B4), si elles confirment la nature laminaire de cette industrie comme son appartenance à l'OIS3, permettent de proposer qu'elle incarne, non pas l'émergence de traditions $L S A$, mais une expression jusqu'alors non documentée en Éthiopie du MSA final. Or, 
cette question de la perduration de traditions techniques ancrées dans le MSA, notamment sous la forme du maintien de méthodes Levallois, est un sujet particulièrement intéressant dans le contexte de l'Afrique orientale (Bon et Fauvelle, 2014).

Dans cette zone d'étude, le LSA proprement dit n'est donc documenté que bien plus tardivement, à la toute fin du Pléistocène, sans que nous puissions apprécier sa genèse ni a fortiori sa transition éventuelle avec le contexte précédemment décrit, puisqu'un très long hiatus documentaire les sépare, correspondant peu ou prou à l'ensemble de l'OIS2. C'est en effet lors de la mise en place de conditions plus humides aux alentours de 12 à $11000 \mathrm{cal} B C$ (B1s4 puis B1s1), que nous retrouvons les traces d'une présence humaine dans la plaine de la Bulbula. Auparavant, les conditions régnant lors de l'OIS2 étaient-elles trop arides pour permettre une installation dans cette zone? L'épisode volcanique dont résulte l'épais dépôt de ponces de l'Abernosa pumice member a-t-il lui aussi contribué à dégrader durablement les conditions de vie dans cette portion du Rift ? Quoi qu'il en soit, à l'instar d'autres secteurs géographiques africains, le «Big Dry » qui marque l'OIS2 s'accompagne ici d'une absence complète de traces d'occupation humaine.

Un même phénomène s'observe au moment du Younger Dryas (circa $11000-9800$ cal BC) : une nouvelle fois, l'occupation humaine paraît s'interrompre durablement, avant que le retour de conditions humides annonçant la transgression majeure des lacs au début de l'Holocène ne s'amorce (DW2s2 puis DW2s1, l'un et l'autre datés du $X^{\mathrm{e}}$ millénaire cal $\mathrm{BC})$. À ces différentes ruptures dans l'occupation humaine à la charnière du Pléistocène et de l'Holocène, répond une forte diversité des expressions et des traditions techniques correspondant à ces différents épisodes, diversité qui tendrait davantage à illustrer l'existence de plusieurs vagues de peuplement que l'évolution sur place d'une seule et même population (Ménard et al., soumis ; Ménard, thèse en cours). De fait, les sites attribués à cette période illustrent une diversité proprement inédite du LSA éthiopien, dont l'expression "classique», dominée par la recherche des célèbres lunates, se révèle n'être alors qu'une composante parmi d'autres (limitée ici à la partie supérieure de la séquence de B1s1) et non un modèle générique. Ces sites permettent aussi d'apporter un nouvel éclairage sur le moment précis où, dans cet environnement progressivement marqué par une plus forte humidité, la chasse aux mammifères terrestres cède au moins partiellement la place à une économie halieutique : d'après les données réunies au Deka Wede, cet épisode se situe dans le courant du $X^{e}$ millénaire, entre l'occupation de DW2s2 et celle de DW2s1. Il s'agit là d'un jalon dont il sera très intéressant d'analyser la portée à une plus grande échelle géographique, en cherchant notamment à confronter ces données avec celles provenant d'autres environnements lacustres d'Afrique de I'Est, en direction du Kenya notamment (pour une synthèse : voir Barham et Mitchell, 2008). Mais il faudra pour cela être en 
mesure d'apprécier plus finement l'évolution du milieu, en cherchant non seulement à décrire la hausse du niveau des lacs mais aussi l'évolution des populations de poissons qu'ils abritent (estimation démographique, éthologie des espèces représentées, etc.), afin d'interpréter plus finement les mutations économiques relevées.

Parmi les questions qui avaient servi de point de départ à notre enquête, était également celle du développement du Néolithique, en particulier de l'adoption d'une économie pastorale. À quand remonte cette pratique, documentée bien plus précocement dans d'autres régions que ce n'est le cas jusqu'à présent dans la partie éthiopienne de la vallée du Rift ? Cette région a-t-elle plus longtemps servi de «zone refuge » à des populations de chasseurs-cueilleurs réfractaires à l'adoption de l'élevage (Gutherz, 2008) ? Ces différentes questions, nous ne sommes malheureusement guère en situation de pouvoir y répondre. Nous pouvons simplement constater l'existence, à une période sans doute bien postérieure à la dernière régression, période que seul le contexte de B1s2 permet d'attribuer à la seconde moitié du $1^{\text {er }}$ millénaire de notre ère, de traces d'installations humaines par des groupes pratiquant une industrie lithique dominée à présent par la recherche d'éclats, associée à l'usage de la céramique (cf. B2 et B5, auxquels s'ajoutent de nombreux indices dispersés en surface de la plaine), mais dont il n'est pas possible de dire si, oui ou non, il s'agit de pasteurs, en dépit de maigres indices livrés en ce sens par B1s2. Même si tel est le cas, ces occupations marquent-elles pour autant le premier étage pastoral de cette partie centrale de la vallée du Rift ? II faudrait, pour répondre à cette question, se hisser au-dessus des eaux du lac et parvenir à mieux documenter la séquence archéologique des premiers millénaires de l'Holocène. Pour l'heure, en dépit de nos recherches sur les reliefs avoisinants (collines de Macho Hill et Waso Hill), comme sur le rocher d'Aga Dima, qui a du alors longtemps fonctionner comme une île ou une presqu'île, ou bien de prospections menées le long des paléo-rivages lacustres perceptibles dans la plaine, et qui se sont toutes révélées négatives, nous n'y sommes pas parvenus. En 2011, nous nous sommes en effet éloignés du canyon de la Bulbula afin de tenter d'appréhender des contextes archéologiques pouvant correspondre à des installations sur les bords du lac lors du dernier épisode de transgression majeure de celui-ci. Malheureusement, le suivi des paléo-rivages n'a pas permis de localiser de telles installations, les conditions environnementales actuelles limitant de fait la possibilité de les déceler. Ainsi, la chronologie précise des occupations de cette région demeure donc pour l'instant bornée aux conditions contemporaines de différentes phases de régression lacustre, lorsque des groupes humains sont venus s'installer aux abords de la rivière Bulbula, ce qui nuit à notre connaissance des phases contemporaines d'une bonne partie de l'Holocène, entre circa 9000 et 3500 cal BC. 
Les recherches à venir doivent justement s'employer à combler ces vides...comme à les confirmer, ce qui serait tout aussi important quoique bien plus difficile. Ainsi, il faudra poursuivre les recherches dans différents contextes géomorphologiques et y multiplier les observations pédosédimentaires comme les datations, recourant à d'autres méthodes que celles que nous avons jusqu'alors employées (OSL sur sédiment, Ar-Ar sur ponces) afin de confirmer la réalité d'une absence d'occupations humaines lors de l'OIS2. II faudra agir de même vis-à-vis de notre enquête sur la fin du Pléistocène et le début de l'Holocène, autour de la question de l'absence de peuplement humain lors du Younger Dryas, comme en continuant à rechercher des contextes archéologiques fiables sur les paléo-rivages, ce que nous ne sommes pas parvenus à faire jusqu'à présent. À tout le moins, désormais, si beaucoup reste à faire, nous pensons avoir franchi une étape dans la précision des questions que nous pensons pouvoir poser à ce paysage et aux hommes qui l'occupèrent, sans doute de façon bien moins pérenne que ne l'implique l'image couramment associée à cette région du monde, celle d'un berceau de l'humanité plusieurs fois renouvelé.

\section{Remerciements}

Nous tenons à remercier la Commission des fouilles du MAE, l'ARCCH (Ethiopian Authority for Research and Conservation of Cultural Heritage) et le CFEE pour leur aide financière, administrative et logistique dans la réalisation de cette mission, ainsi que I'INRAP et I'UMR 5608 TRACES (Université de Toulouse - le Mirail) pour leur soutien conjoint. Merci également au rapporteur anonyme de cet article pour ses conseils et corrections. 


\section{Annexe 1 : Descriptifs des secteurs fouillés dans la plaine de la Bulbula}

Nos fouilles dans la plaine de la Bulbula ont porté sur l'exploration de dix sites: quatre d'entre eux sont localisés à Bulbula 1 (B1s1, s2, s3 et s4), trois aux environs de ce dernier (B2, B4 et B5) et trois au Deka Wede (DW1, DW2s1 et s2) ${ }^{15}$. À ces différents sites dont le détail est donné ciaprès, s'ajoute le fruit de prospections de surface conduites à Bulbula 1 en 2008 (thalweg 1 à 3 ; échantillons \#4000 à 4002) et dans les environs de ce secteur au cours des deux campagnes suivantes (échantillons \#9000 à 9022 et \#9023-9025). Les différents corpus archéologiques issus de ces recherches ont été déposés au Musée National à Addis-Abeba ${ }^{16}$.

\section{Bulbula 1 secteur 1 (B1s1)}

Années d'intervention : 2008, 2009 et 2010

Topographie du secteur, conditions d'observation : B1s1 est localisé sur le flanc nord de la dépression de Bulbula 1, implantée en rive droite de la rivière Bulbula. Plus précisément, ce site occupe un petit replat $(9 \times 6$ mètres de dimensions maximales) en contrebas d'une corniche peu élevée ( 2 mètres d'élévation environ) ménagée par l'érosion de la formation lacustre indurée baptisée «dalle ». Ce replat s'étale entre la corniche et une ligne d'effondrement de gros blocs qui en sont issus. Si le site doit sa conservation à cette disposition particulière, il a toutefois été entaillé par un chenal temporaire («thalweg 3 » ou B1t3), créant une coupe naturelle qui révéla l'existence d'un dépôt archéologique à cet endroit.

Coordonnées GPS : N 07²9.118' / E 03841.905'

Historique et nature de l'intervention: L'exploration de ce secteur découvert puis sondé en 2008 s'est poursuivie au cours des deux campagnes suivantes en 2009 et 2010 . L'opération a concerné la fouille totale ou partielle de $6 \mathrm{~m} 2$ (slaa, sla, slb, slc, sld, sle), composant une tranchée perpendiculaire à la corniche d'environ 5 mètres d'extension dans l'axe WNW/ESE (Fig. 7). Cette opération a révélé une séquence livrant

\footnotetext{
${ }^{15}$ Bulbula 3 désigne une coupe naturelle observée en rive gauche de la Bulbula, en face de B1, où un artefact lithique isolé a été observé en 2008, la campagne suivante n'ayant en définitive pas permis d'identifier de niveau archéologique à cet endroit.

16 Des prélèvements sédimentaires (\#10015-10024 et \#10038-10076), des blocs orientés destinés à une étude micromorphologique (\#10001-10004 et \#10034-10037), divers échantillons destinés à effectuer des datations selon différentes méthodes (échantillons $\# 10025$ à 10033, \#10077-10086, auxquels s'ajoutent une dizaine d'échantillons en 2008 et 8 en 2011) ainsi que des analyses palynologiques (\#10005-10014) et de phytolithes (2 échantillons en 2011) ont été exportés vers la France avec l'autorisation des autorités éthiopiennes. À cela, s'ajoute l'exportation temporaire de 108 pièces lithiques destinées à une analyse géochimique, ainsi qu'à un test tracéologique effectué par Laurence Astruc. Des échantillons d'ossements de poissons $(n=341)$ issus de DW2s1 ont également été temporairement exportés en France afin de parfaire leur détermination grâce à l'autorisation du $\mathrm{ARCCH}$.
} 
6 niveaux archéologiques principaux, formant deux ensembles (VI-VII-VIIIIX et XI-XII) séparés l'un de l'autre par un horizon détritique (X), le tout composant environ 1,60 mètres d'épaisseur. Par ailleurs, profitant de l'érosion due au chenal traversant cette zone (B1t3), une coupe perpendiculaire à l'axe de cette tranchée a été aménagée afin de suivre l'extension latérale de ces formations vers le SSW. Outre la collecte du mobilier archéologique par passe au sein de chaque US à l'aide d'un tamisage systématique, nous avons opéré le prélèvement de six blocs orientés, destinés à une analyse micromorphologique, ainsi que de plusieurs prélèvements sédimentaires.

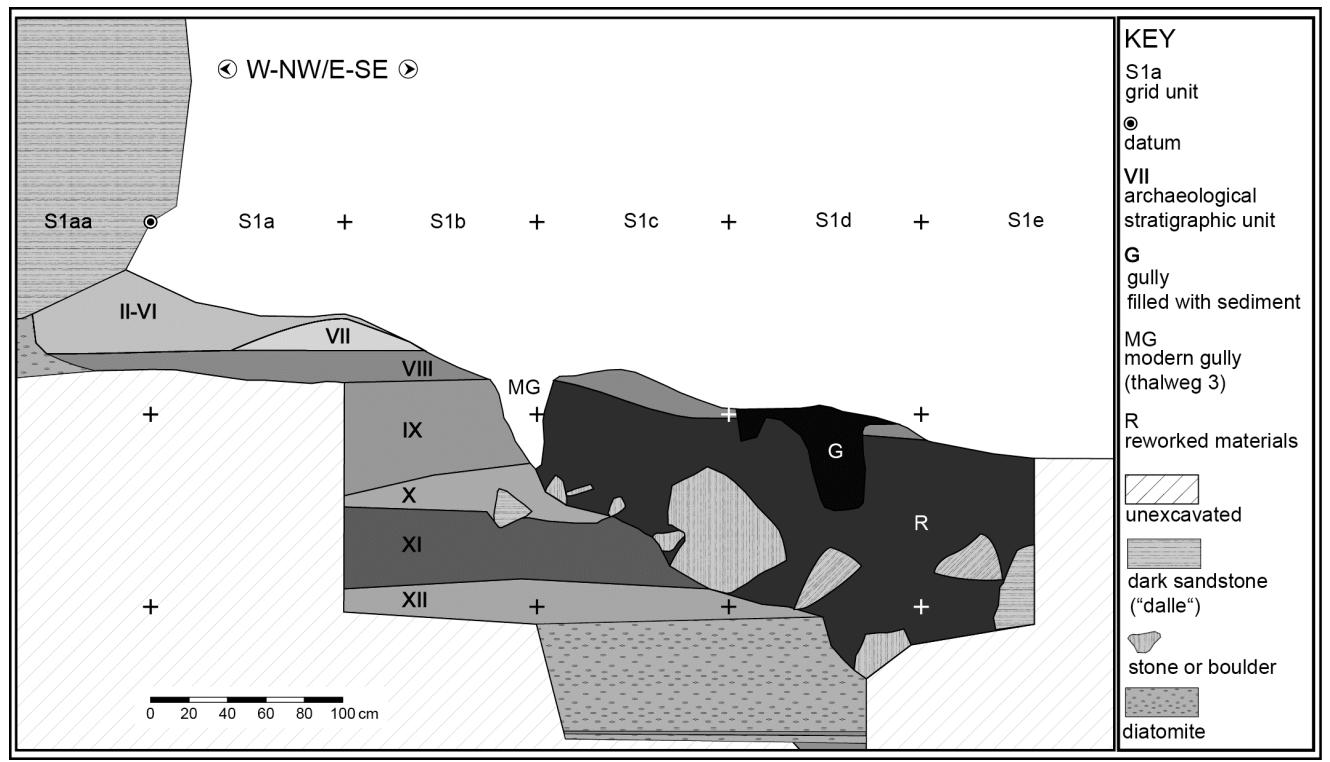

Fig. 7 - Coupe sagittale du site B1s1 (DA0 : C. Ménard).

\section{Description stratigraphique et reconstitution paléotopogra-phique}

(cf. $\mathbf{n}^{\circ} \mathbf{5}$ Fig. 5) : Nous pouvons considérer que les occupants du site ont profité de la protection offerte par la petite corniche au pied duquel il est implanté, qui dominait alors la rivière dont le niveau était plus élevé qu'il ne l'est aujourd'hui. Sans doute la dépression de Bulbula 1 constituait-elle alors un fond de ria correspondant à une transgression mineure des eaux du lac Abijata, où se jetait la rivière Bulbula. La séquence enregistrée est en effet marquée par l'alternance de dépôts d'origine alluviale et/ou lacustre avec de véritables sols d'occupation préhistorique, auxquels s'ajoute l'épisode détritique consécutif à l'érosion de la corniche susjacente (US X). Les dates radiocarbones obtenues, très resserrées bien qu'appartenant aux deux extrémités de la séquence archéologique, sont en accord avec une sédimentation rapide ayant permis de sceller régulièrement l'empilement des sols d'occupation. 
Datation radiocarbone : $11480 \pm 60$ BP (Ly-6059) soit 13 468-13 197 cal BP ou 11 471-11 287 cal BC, datation AMS sur charbon de bois (niveau VIII ; à cela s'ajoute le résultat non valide d'un charbon de bois provenant du même ensemble) ; $11480 \pm 50$ BP (Beta-292524) soit 13 460-13 221 cal BP ou 11 460-11 300 cal BC, datation AMS sur charbon de bois (niveau XIV, équivalent niveau XII ; échantillon \#10079).

Nature du mobilier archéologique: Les différents niveaux archéologiques livrent une très grande densité de mobilier, conjuguant industrie lithique (plusieurs dizaines de milliers de vestiges), restes osseux (plus de 19000 éléments décomptés), fragments d'œuf d'autruche (dont certains transformés en perles) et nombreux charbons. Le tamisage des sédiments a permis de montrer que toutes les fractions dimensionnelles sont représentées et que ce matériel peut être considéré de ce point de vue comme intègre, ce que confirme l'état de fraîcheur des pièces lithiques. Les ossements possèdent en revanche des surfaces altérées, mais les stigmates observés traduisent l'action de processus chimiques (dus à l'acidité des sols ou à la présence de racines) et non mécaniques (transport). L'ensemble de cette séquence se rapporte au LSA.

Échantillonnage archéologique : \#1001 à 1016 (2008), \#1017 à 1066 (2009) et \#1067 à 1073 (2010).

\section{Bulbula 1 secteur 2 (B1s2)}

Années d'intervention : 2008 et 2009

Topographie du secteur, conditions d'observation: $\grave{A}$ quelques mètres en contrebas de B1s1, se forme un léger palier (au sommet des dépôts volcano-clastiques) où se rejoignent les différents chenaux (thalwegs 1, 2 et 3 ) qui traversent la dépression de Bulbula 1. Plus précisément, les chenaux B1t2 et B1t3 se rejoignent pour ne former ensuite qu'un seul et unique chenal, tandis que le thalweg B1t1, localisé au sud-est des précédents, demeure indépendant et incise profondément les formations colluviales et alluviales déposées dans le fonds de la dépression de Bulbula 1, créant une coupe naturelle permettant d'observer la séquence décrite ci-après (bordure droite de B1t1).

Coordonnées GPS : N 07²49.103' / E 3841.912'

Historique et nature de l'intervention : Notre intervention a consisté à rectifier cette coupe naturelle sur une longueur d'environ 3,50 mètres et une hauteur de 2,50 mètres (2008), puis à fouiller un $\mathrm{m} 2$, baptisé $\mathrm{s} 2 \mathrm{a}$, dont seule la moitié SE a été décapée et tamisée systématiquement sur toute la puissance stratigraphique relevée à cet endroit, soit 2,05 mètres de haut (2009).

Description stratigraphique et reconstitution paléotopogra-phique (cf. $\mathbf{n}^{\circ} \mathbf{9}$ Fig. 5) : Du haut vers le bas : socle de limons indurés (épaisseur 
inconnue), formation alluviale («Alluvial fin », «Alluvial graveleux »; ép. : 0,65 mètre) recouverte par des colluvions (ép. : 1,40 mètres). Au sein de cette formation colluviale, alternance de dépôts fins (SDF1 à 4) et d'un horizon de ponces colluviées (PC), entrecoupés par deux paléosols (PS1 et PS2), le tout étant coiffé par le sol actuel (S3), ce qui donne la succession suivante (du bas vers le haut) : SDF1/PC/PS1/SDF2-3/PS2/SDF4/S3. Deux horizons contiennent du matériel archéologique : Alluvial graveleux (1-2) et PS2. Le niveau d'occupation associé à PS2 semble correspondre à une installation humaine in situ dans le fond de la dépression de Bulbula 1, postérieurement à la dernière régression lacustre, ce que confirme la nature du mobilier comme la date obtenue.

Datation radiocarbone : Un charbon de bois prélevé en 2008 (\#3) dans le PS2 a livré la date de $1330 \pm 30$ BP, soit 652-766 cal AD (Ly-6060). Tentative avortée de datation d'un os issu de l'alluvial graveleux (absence de collagène) puis résultat non valide d'un charbon de bois provenant du même ensemble.

Nature du mobilier archéologique : La formation alluviale de base comporte de nombreux vestiges archéologiques (vestiges lithiques taillés, fragments d'os) qui résultent de la remobilisation de niveaux LSA d'après une industrie dominée par la production de lames et de lamelles (s'agit-il de la remobilisation de niveaux contemporains de B1s1 ?). Le mobilier de PS2 relève en revanche d'une tradition technique radicalement différente, dominée par la recherche d'éclats, où l'industrie en obsidienne taillée (36 pièces) est rejointe par des tessons de céramique (3). Parmi la faune (une centaine de restes), on note la présence hypothétique d'un reste de bœuf (Bos taurus), mais les surfaces osseuses sont trop corrodées pour confirmer cette identification.

Échantillonnage archéologique : \#3000 et 3001 (2008) et \#3002 à 3012 (2009).

\section{Bulbula 1 secteur 3 (B1s3)}

Années d'intervention : 2009 et 2010

Topographie du secteur, conditions d'observation : À proximité de la dépression de Bulbula 1 et toujours en rive droite de la rivière, l'érosion occasionnée par le tracé d'un petit chenal temporaire collectant les eaux de surface, a isolé une petite butte d'environ 2 à 3 mètres de haut entre celui-ci et la berge verticale de la Bulbula.

Coordonnées GPS : N 0749.153' / E 03841.863'

Historique et nature de I'intervention : À la suite des observations conduites à Bulbula 4 relatives à la présence résiduelle d'un paléosol ancien contenant des industries sur le rebord du canyon de la Bulbula (cf. ci-dessous), une prospection systématique en rive droite de celle-ci nous a 
amené à identifier cette même formation à $B 1 s 3$, en association de nouveau avec des vestiges lithiques. La fouille de ce secteur débuta en 2009 et fut poursuivie en 2010. Elle concerna au total $6 \mathrm{~m}^{2}$ (s3a à s3f), qui firent l'objet d'un décapage en suivi stratigraphique et par passe, accompagné d'un tamisage systématique. Plusieurs prélèvements de sédiments furent également effectués (\#10045 à 10048).

\section{Description stratigraphique et reconstitution paléotopogra-phique} (cf. $\mathbf{n}^{\circ} \mathbf{2}$ Fig. 5) : Le mobilier archéologique est contenu dans la partie inférieure d'un palésol brun clair d'environ 1,30 mètre d'épaisseur, reposant sur une surface irrégulière correspondant à l'altération d'un paléosol plus ancien, fortement induré, et qui repose lui au contact de la « dalle». Le paléosol brun clair est manifestement le fruit d'une histoire complexe: il est notamment divisé par la présence d'un fin niveau de ponces, qui marque la limite entre une partie supérieure meuble et une partie inférieure plus encroûtée. Cette limite pédo-sédimentaire est également archéologique: la partie supérieure du paléosol est stérile (note: un contrôle mériterait d'être effectué dans sa partie sommitale), I'industrie étant contenu dans la partie inférieure. Dans cette dernière (ép. : 0,50 mètre), le mobilier est davantage concentré à la base, au contact du paléosol ancien induré, en particulier en ce qui concerne la fraction fine. L'ensemble de ce paléosol est coiffé par un niveau de ponces, qui pourrait correspondre à un vestige de l'Abernosa pumice member.

Datation radiocarbone : non

Nature du mobilier archéologique: II est presque exclusivement composé de vestiges lithiques ( 1127 pièces d'une dimension supérieure à $1 \mathrm{~cm}$ ), les restes osseux se limitant un fragment d'humérus d'hippopotame. II est vraisemblable que ce mobilier rassemble des vestiges appartenant à plusieurs occupations. Toutefois, l'industrie concernée semble homogène du point de vue technologique et relever d'une même tradition technique, appartenant au MSA final.

Échantillonnage archéologique : \#7000 à 7016 (2009) et \#7017 à 7039 (2010).

\section{Bulbula 1 secteur 4 (B1s4)}

Années d'intervention : 2009 et 2010

Topographie du secteur, conditions d'observation : Comme B1sl qui se trouve en contrebas de lui, B1s4 occupe le pourtour nord de la dépression de Bulbula 1, à la gauche du sentier qui mène à la rivière. Le talus de ce sentier offre une coupe naturelle façonnée par l'érosion, qui a permis d'identifier le dépôt archéologique concerné par ce site.

Coordonnées GPS : N 0749.125' / E 03841.909' 
Historique et nature de l'intervention : Reconnu dès 2008, ce secteur fit l'objet de deux tranchées perpendiculaires au sentier l'année suivante ( $T 1$ et $T 2$ ), seule la première (T1) donnant lieu à une fouille poursuivie en 2010, laquelle concerna au total $4 \mathrm{~m}^{2}$ (numérotés A à C). En 2010, l'observation de plusieurs coupes naturelles, situées aux alentours de B1s4, a permis de replacer la stratigraphie de ce secteur dans la séquence générale environnante. Outre sa fouille effectuée selon le protocole habituel (fouille par passe selon délimitation stratigraphique, tamisage systématique), B1s4 fit l'objet de deux prélèvements de blocs orientés destinés à une analyse micromorphologique (\#10036 et 10037) ainsi que de plusieurs échantillons sédimentaires (\#10041 à 10044).

\section{Description stratigraphique et reconstitution paléotopogra-phique} (cf. $\mathbf{n}^{\circ} \mathbf{4}$ Fig. 5) : La stratigraphie de B1s4 se compose de deux paléosols séparés l'un de l'autre par un dépôt de limons massifs, lié à une transgression mineure des eaux du lac. Le paléosol inférieur (ou 1) s'est développé aux dépends de colluvions remaniant des sédiments d'origine volcanoclastiques; le second (ou 2) s'est développé sur les limons intermédiaires. Cette séquence possède une épaisseur totale de 1,10 mètres, dont 0,65 mètre pour le paléosol $1,0,30$ mètre pour les limons et 0,15 mètre pour le paléosol 2. Cette séquence repose sur des colluvions stériles (limons dégradés) situés au contact de la « dalle » et est coiffée par un dépôt de ponces (environ 0,50 mètre à cet endroit), que recouvre ici l'épais dépôt de limons correspondant à la transgression majeure holocène. La seule date dont nous disposons concerne le paléosol inférieur, et il est impossible d'apprécier l'écart temporel entre les occupations contemporaines de ce dernier et celles contenues dans le paléosol supérieur, dont nous pouvons seulement conclure qu'elles sont antérieures à la grande transgression holocène. En d'autres termes, si l'on suit la date obtenue pour le paléosol inférieur, ce dernier est antérieur à la mise en place de la séquence voisine de B1s1; en revanche, le paléosol supérieur peut lui être postérieur.

Datation radiocarbone : $12040 \pm 50 \mathrm{BP}$ (Beta-332588) soit 12 040-11 840 cal BC, datation AMS sur charbon de bois (Paléosol inférieur ou 2).

Nature du mobilier archéologique: Le corpus livré par le paléosol inférieur est numériquement beaucoup plus riche que celui du paléosol supérieur. L'un et l'autre sont dominés par les vestiges lithiques, les restes osseux étant beaucoup plus rares (105 restes en tout). Plus précisément, le décompte de l'ensemble du mobilier lithique du paléosol inférieur (carrés A, B, C et D, fouilles 2010), toutes fractions confondues, s'élève à 1858 pièces lithiques ; celui du paléosol supérieur (fouilles 2009 et 2010), toutes fractions confondues, rassemble seulement 345 pièces lithiques. II s'agit dans les deux cas d'une industrie dominée par la recherche de lames et de lamelles, appartenant sans conteste au LSA. 
Échantillonnage archéologique : \#8000 à 8009 (2009) et \#8010 à 8026 (2010).

\section{Bulbula 2 (B2)}

Année d'intervention : 2008.

Topographie du secteur, conditions d'observation: $\grave{A}$ quelque distance au sud-ouest de Bulbula 1, toujours sur la rive droite de la rivière, un sondage a été exécuté sur un léger relief dominant la berge raide de celle-ci.

\section{Coordonnées GPS : N 07049.095' / E 38²41.721'}

Historique et nature de l'intervention : L'emplacement de ce sondage a été guidé par la reconnaissance, à la surface du sol de ce léger relief, d'un mobilier composé de vestiges lithiques (obsidienne taillée) associés à des tessons de céramiques. Le sondage a pris la forme d'une tranchée perpendiculaire à la pente sur le rebord sud-ouest de ce léger relief, de 2 $\mathrm{m}$ de long et de 0,50 mètre de large. Cette tranchée a été subdivisée en deux secteurs d'un mètre d'extension chacun, correspondant respectivement aux carrés $A$ et $B$. Situé en amont, le carré $A$ n'a été sondé que jusqu'à une profondeur de $25 \mathrm{~cm}$, l'exploration en aval du carré B ayant été poursuivie jusqu'à un mètre.

\section{Description stratigraphique et reconstitution paléotopogra-phique} (cf. $\mathbf{n}^{\circ} 8$ Fig. 5) : La stratigraphie identifiée dans le carré B est la suivante, du haut vers le bas : sol végétal actuel (ép. : $0,05 \mathrm{~m}$ ) ; sable gris comportant des nodules de ponces dispersés et des granules d'obsidienne roulées (ép. : 0,45 m) ; ponces colluviées (ép. : 0,10 m) ; sable jaune-brun (ép. : $0,25 \mathrm{~m}$ ); au-delà de $-85 \mathrm{~cm}$ de la surface, présence de blocs de limons emballés dans le même sédiment sableux jaune-brun (la base de ce niveau n'a pas été atteinte).

Datation radiocarbone : Tentative avortée de datation d'un os issu de la couche de sable jaune-brun (absence de collagène) puis résultat non valide d'un charbon de bois provenant du même ensemble.

Nature du mobilier archéologique: L'industrie lithique recueillie en surface relève exclusivement d'un débitage d'éclats, auquel sont associés des éclats retouchés et des grattoirs. On note également la présence de tessons de céramique. Un assemblage identique provient de la couche sommitale de sable gris observée dans le sondage, qui a livré d'assez nombreux vestiges fauniques et lithiques, constitués par des débitages d'éclats, associé à un grattoir. La présence d'un tesson confirme également la grande similitude de ce mobilier avec celui recueilli en surface. La couche inférieure de sables jaune-brun contient quelques vestiges fauniques dont des vertèbres de poisson, d'assez nombreux coquillages et de rares micro-vestiges lithiques en obsidienne. D'une façon générale, les 
vestiges osseux sont mal conservés comme en témoigne leur fragmentation et l'altération de leurs surfaces. Toutefois, plusieurs taxons ont été reconnus provenant des niveaux compris entre 20 et $70 \mathrm{~cm}$. Le spectre comprend un grand bovidé (format buffle), un bovidé de taille 3 (format grande gazelle), du Tilapinii et du cercopithèque.

Échantillonnage archéologique : \#2001 à 2009 (2008)

\section{Bulbula 4 (B4)}

Années d'intervention : 2008 et 2009

Topographie du secteur, conditions d'observation : Localisé lui aussi en rive droite de la Bulbula, B4 correspond à un petit relief implanté au sommet de sa berge raide.

Coordonnées GPS : N $07^{\circ} 48.966^{\prime}$ / E 38²41.627'

Historique et nature de l'intervention : Reconnu en prospection dès 2008, B4 fit l'objet d'un sondage de $1 \mathrm{~m} 2$ en 2009, qui permit de lever une séquence d'environ 0,6 mètre de haut.

Description stratigraphique et reconstitution paléotopogra-phique (cf. $\mathbf{n}^{\circ} 1$ Fig. 5) : B4 occupe une position stratigraphique et paléotopographique similaire à celle de B1s3. II s'agit en l'occurrence d'une petite butte résiduelle d'un paléosol ancien reposant sur la «dalle». Plus précisément, le mobilier archéologique se concentre sous la forme de poches à la base de ce paléosol brun clair (épaisseur de ce dernier : 0,45 mètre), au contact d'un sol induré plus ancien (ép. : 0,20 mètre) reposant sur la « dalle».

Datation radiocarbone: Non.

Nature du mobilier archéologique : II se compose principalement de vestiges lithiques, auxquels sont associés quelques rares fragments d'os. L'industrie appartient au MSA final.

Échantillonnage archéologique : \#5000 à 5004 (2009)

\section{Bulbula 5 (B5)}

Année d'intervention : 2009

Topographie du secteur, conditions d'observation: Le sondage baptisé $\mathrm{B} 5$ a été implanté en reculant l'une des coupes naturelles creusées sur les bords des nombreux chenaux temporaires contemporains que compte la rive droite de la Bulbula à l'endroit où se développe le Gilbert delta. Ces coupes avaient en effet permis d'observer des séquences comportant quelques vestiges (os, éléments lithiques, tessons de céramique) disséminés dans le sol formé après la dernière régression lacustre.

Coordonnées GPS : N $07^{\circ} 49.269^{\prime}$ / E 3842.423' 
Historique et nature de l'intervention : Dans cette zone prospectée en 2009, un sondage de $2 \mathrm{~m}^{2}$ fut ouvert la même année. II permit de préciser une séquence supérieure d'environ 1,40 mètre, sans parvenir à identifier de niveau(x) archéologique(s) justifiant la poursuite de l'exploration de cette zone.

Description stratigraphique et reconstitution paléotopographique : la stratigraphie relevée dans cette zone comporte deux ensembles principaux: à la base, un épais dépôt de sables correspond au fonctionnement alluvial du Gilbert delta; au-dessus, suite à la dernière régression, s'est formé un sol au dépend de ces sables et de colluvions remaniant des sédiments d'origine volcano-clastiques d'une épaisseur approximative de l'ordre de 1,40 mètres. Plus précisément, cette séquence supérieure se décompose en un paléosol (ép.: environ 0,80 mètre), surmonté par un lit de ponces discontinu (ép. : 0,20 mètre), coiffé par le sol actuel (ép.: 0,40 mètre). Le paléosol contient quelques vestiges archéologiques épars, concentré davantage dans sa partie supérieure, au contact des ponces (quelques obsidiennes taillées et quelques tessons de céramique).

Datation radiocarbone : Non. Nous pouvons simplement apprécier le fait que cette séquence supérieure s'est développée postérieurement à la dernière régression et au Gilbert delta qui la précède, lequel a pu être daté de $4800 \pm 30$ BP (Beta-295900), soit 3650-3520 BC, datation obtenue par AMS sur un coquillage prélevé dans les environs de ce secteur.

Nature du mobilier archéologique : Rares vestiges lithiques taillés et tessons de céramique.

Échantillonnage archéologique : \#6000 à 6005 (2009).

\section{Deka Wede 1 (DW1)}

Année d'intervention : 2010

Topographie du secteur, conditions d'observation : DW1 est le nom donné à un site visible dans une coupe naturelle crée par le tracé du Deka Wede, affluent temporaire de la rivière Bulbula puisant sa source sur le flanc occidental du volcan Alutu. À cet endroit, les berges du Deka Wede se présentent sous la forme de coupes naturelles verticales très élevées. L'horizon archéologique a pu être observé en 2010 à la faveur d'un effondrement ponctuel intervenu à la base de cette coupe instable, localisée en rive droite.

Coordonnées GPS : N $07^{\circ} 47.570^{\prime} /$ E $38^{\circ} 41.469^{\prime}$

Historique et nature de l'intervention: DW1 fut préalablement identifié par Street qui décrit, au sein de l'une de ses coupes géologiques de référence, un paléosol daté de $27050 \pm 1540$ BP (SUA-588; Street, 1979 ) ayant livré une industrie lithique qualifiée par elle, sur la foi d'une 
observation menée par Brandt, Clark et Kurashina de «Early Late Stone Age » grâce à sa composante laminaire (conclusions reproduites notamment par Brandt, 1986). Notre propre intervention s'est bornée à un rafraîchissement de coupe naturelle, étant entendu que toute fouille extensive est exclue ici : le niveau archéologique est surmonté par une trop grande puissance sédimentaire pour pouvoir être apprécié en planimétrie. Notre principale intervention a donc consisté en un prélèvement ponctuel de sédiment sur 1,60 mètres de longueur de coupe et seulement 0,15 mètre de profondeur (échantillon \#13000) à l'endroit où le paléosol contenant le mobilier archéologique était le mieux exprimé et le plus accessible (épaisseur à cet endroit : 0,30 mètre; paléosol noté PS5 dans notre propre nomenclature de la séquence pédo-sédimentaire relevée dans le secteur du Deka Wede). Le suivi latéral de cet horizon pédologique a permis de recueillir à quelques distances, de part et d'autres de ce point, quelques objets lithiques (\#13001; environ 6 mètres au NE) ainsi qu'un charbon de bois et un os (\#10086; environ 14 mètres au SW).

Description stratigraphique et reconstitution paléotopogra-phique (cf. $\mathbf{n}^{\circ} \mathbf{3}$ Fig. 5) : Le mobilier archéologique est contenu dans un paléosol discontinu développé aux dépends d'une formation alluviale (alternant fraction grossière à la base et fine au sommet) surmontée par un dépôt de limons traduisant une transgression mineure des lacs. II semble que ce paléosol se soit développé sur une petite barre de chenal. Le mobilier était concentré au sommet du paléosol, à l'interface avec les limons susjacents, seuls des vestiges lithiques appartenant à la fraction fine ayant été recueillis dans l'épaisseur de celui-ci.

Datation radiocarbone : $29120 \pm 160$ BP (Beta-292527) soit 34 497-33 262 cal BP, datation AMS sur charbon de bois (échantillon \# 10086).

Nature du mobilier archéologique : Vestiges lithiques (251 pièces) et osseux. Un charbon de bois a été recueilli à quelques distances dans la même formation (échantillon \#10086). Ce mobilier appartient au MSA final.

Échantillonnage archéologique : \#13000, \#13001 (quelques vestiges lithiques prélevés en coupe), \#10086 (charbon prélevé en coupe).

\section{Deka Wede 2 secteur 1 (DW2s1)}

Années d'intervention : 2010 et 2011

Topographie du secteur, conditions d'observation : $\grave{A}$ l'instar de DW1, DW2s1 est un site révélé par l'érosion active des berges du Deka Wede, en l'occurrence en rive gauche de celui-ci. Toutefois, sa position topographique et stratigraphique a permis d'intervenir sur une surface plus conséquente que ce n'est le cas à DW1. 


\section{Coordonnées GPS : N $07^{\circ} 47.519^{\prime}$ / E 38 $41.520^{\prime}$}

Historique et nature de l'intervention : La fouille de ce site découvert et sondé une première fois en 2010 a été poursuivie l'année suivante. Au total, ces recherches ont permis de procéder à l'exploration d'une surface de l'ordre de $4 \mathrm{~m}^{2}$ du paléosol le plus récent (si l'on exclut le sol actuel) observé dans le secteur du Deka Wede. Ce paléosol noté PS1 dans notre propre nomenclature contient ici un abondant mobilier archéologique. Prélèvement d'un échantillon de sédiment du PS1 (\#10049; 2010).

\section{Description stratigraphique et reconstitution paléotopogra-phique} (cf. $\mathbf{n}^{\circ} \mathbf{7}$ Fig. 5) : S'il est aujourd'hui perché à plusieurs mètres au dessus du fond du canyon du Deka Wede, DW2s1 devait au contraire occuper le fond d'un petit vallon lors de l'occupation humaine, tandis que la rivière coulait plus haut que ce n'est le cas aujourd'hui. Le niveau archéologique de DW2s1 est situé dans la partie sommitale d'un ensemble pédosédimentaire composé par l'alternance de colluvions et d'alluvions remobilisant des sédiments d'origine volcano-clastiques (ponces, etc.), de limons traduisant des transgressions mineures du niveau des lacs, le tout entrecoupé de plusieurs paléosols notés PS1 à PS4. En l'occurrence, le paléosol noté PS1 se développe au sommet d'une formation colluviale contenant une succession de paléosols plus anciens (PS3 et PS2) traduisant donc l'existence d'une alternance entre phases de stabilité du paysage (au cours desquelles se développe une végétation entraînant la formation de ces sols), et des épisodes érosifs (amenant leur recouvrement par des colluvions). Cet ensemble colluvial est encadré par des dépôts alluviaux et/ou lacustres : en dessous, des limons correspondent à une remontée mineure du niveau du lac Abijata; au dessus, un puissant ensemble sédimentaire composé de sables et de limons correspond en revanche à la transgression majeure holocène entraînant la formation du «macrolake » (cf. « synthem 3 »).

Datation radiocarbone : $9830 \pm 50$ BP soit 9350-9240 cal BC (Beta320183), datation AMS sur charbon de bois (échantillon \#11011).

Nature du mobilier archéologique : Vestiges lithiques (1030 pièces) et osseux (565 restes, dont 341 de poissons), coquillage (4), charbons de bois, appartenant au LSA.

Échantillonnage archéologique : \#11000 à 11003 (2010) et 11004 à 11008 (2011) correspondant à du sédiment prélevé dans le PS1. À cela, s'ajoutent des prélèvements de charbons de bois provenant également de PS1 (\#11010 et \#11011) et du matériel de surface (\#11009).

\section{Deka Wede 2 secteur 2 (DW2s2)}

Années d'intervention : 2010 et 2011 
Topographie du secteur, conditions d'observation : Localisé à faible distance vers le nord-ouest de DW2s1, et visible comme ce dernier dans les coupes naturelles de la rive gauche du Deka Wede, son implantation topographique a seulement permis d'ouvrir une surface de l'ordre de $2 \mathrm{~m}^{2}$.

\section{Coordonnées GPS : N $07^{\circ} 47.528^{\prime}$ / E $38^{\circ} 41.513^{\prime}$}

Historique et nature de l'intervention: L'intervention sur ce site découvert et sondé une première fois en 2010 s'est poursuivie en 2011. Prélèvement d'un échantillon de sédiment du PS4 (\#10050; 2010).

Description stratigraphique et reconstitution paléotopogra-phique (cf. $\mathbf{n}^{\circ} 6$ Fig. 5) : Le niveau archéologique de DW2s2 est situé dans la partie inférieure du même grand ensemble pédo-sédimentaire contenant à son sommet le paléosol PS1 où se situe DW2s1. En l'occurrence, DW2s2 est contenu dans le paléosol noté PS4, lequel s'est plus précisément développé au sein d'une formation colluviale polycyclique attestant une succession de phases érosives entrecoupées d'une meilleure stabilité du paysage accompagnant le développement d'une végétation dont procède la formation de ce sol. Cette formation colluviale est encadrée par une formation alluviale sous-jacente (l'une et l'autre étant séparée par la troncature d'un épisode érosif) et, au dessus, par le dépôt de limons observé sous la formation colluviale contenant les paléosols PS1, PS2 et PS3 (cf. DW1s1). Le paléosol PS4, remarquablement bien exprimé, se développe sur une épaisseur de l'ordre de $10 \mathrm{~cm}$.

Datation radiocarbone : $10040 \pm 50$ BP soit 9820-9360 cal BC (Beta295898), datation AMS sur sédiment humique (échantillon \#10050).

Nature du mobilier archéologique : Vestiges lithiques (1131 pièces) et osseux (4964 restes), fragments d'œufs d'autruche (16) et coquillages (17), charbons de bois, appartenant au LSA.

Échantillonnage archéologique : \#12000 et \#12001 (2010); \#12002 et \#12004 à \#12008 (2011). À cela, s'ajoutent des échantillons appartenant également au PS4 extraits du rafraîchissement de la coupe (\#12003 et \#12009) ainsi que des prélèvements de charbons issu de PS4 (\#12010 et $\# 12011$ ). 


\section{Annexe 2 : Tableau récapitulatif des datations radiocarbones obtenues dans la plaine de la Bulbula}

\begin{tabular}{|c|c|c|c|c|c|c|c|c|c|}
\hline Mission & Labo & $\begin{array}{c}\mathrm{N}^{\circ} \\
\text { labo. }\end{array}$ & $\begin{array}{l}\text { Secteur } \\
\text { Couche }\end{array}$ & Nature & $\begin{array}{c}\mathrm{N}^{\circ} \\
\text { échant. }\end{array}$ & Méth. & $\begin{array}{c}\text { Âge } \\
\text { mesuré } \\
\text { BP }\end{array}$ & $\begin{array}{c}\text { Âge } \\
\text { convent. } \\
\text { BP }\end{array}$ & $\begin{array}{c}\text { Âge } \\
\text { calibré }\end{array}$ \\
\hline \multicolumn{10}{|c|}{ Échantillons archéologiques } \\
\hline $\begin{array}{l}\text { Ziway } \\
2008\end{array}$ & Lyon & 6060 & $\begin{array}{l}\text { B1s2 } \\
\text { PS2 }\end{array}$ & Charbon & 3 & AMS & $\begin{array}{c}1330 \\
\pm 30 \mathrm{BP}\end{array}$ & & $\begin{array}{c}652- \\
766 \mathrm{AD}\end{array}$ \\
\hline $\begin{array}{l}\text { Ziway } \\
2011\end{array}$ & Beta & 320183 & $\begin{array}{l}\text { DW2s1 } \\
\text { PS1 }\end{array}$ & Charbon & 11011 & AMS & $\begin{array}{c}9860 \\
\pm 50 \mathrm{BP}\end{array}$ & $\begin{array}{c}9830 \\
\pm 50 \mathrm{BP}\end{array}$ & $\begin{array}{c}9350- \\
9240 \\
\text { BC }\end{array}$ \\
\hline $\begin{array}{l}\text { Ziway } \\
2010\end{array}$ & Beta & 295898 & $\begin{array}{l}\text { DW2s2 } \\
\text { PS4 }\end{array}$ & $\begin{array}{l}\text { Sédiment } \\
\text { humique }\end{array}$ & 10050 & AMS & $\begin{array}{c}9940 \\
\pm 50 \mathrm{BP}\end{array}$ & $\begin{array}{l}10040 \\
\pm 50 \mathrm{BP}\end{array}$ & $\begin{array}{c}9820- \\
9360 \\
\text { BC }\end{array}$ \\
\hline $\begin{array}{l}\text { Ziway } \\
2008\end{array}$ & Lyon & 6059 & $\begin{array}{l}\text { B1s1 } \\
\text { C.VIII }\end{array}$ & Charbon & 2 & AMS & $\begin{array}{l}11480 \\
\pm 60 \mathrm{BP}\end{array}$ & & $\begin{array}{c}11471- \\
11287 \\
B C\end{array}$ \\
\hline $\begin{array}{l}\text { Ziway } \\
2010\end{array}$ & Beta & 292524 & $\begin{array}{l}\text { B1s1 } \\
\text { c. XIV }\end{array}$ & Charbon & 10079 & AMS & $\begin{array}{l}11490 \\
\pm 50 \mathrm{BP}\end{array}$ & $\begin{array}{l}11480 \\
\pm 50 \mathrm{BP}\end{array}$ & $\begin{array}{c}11460- \\
11300 \\
B C\end{array}$ \\
\hline $\begin{array}{l}\text { Ziway } \\
2008\end{array}$ & Beta & 332588 & $\begin{array}{c}\text { B1s4 } \\
\text { Paleosol } \\
\text { inf. }\end{array}$ & Charbon & 8007 & AMS & $\begin{array}{c}12040 \\
\pm 50 \mathrm{BP}\end{array}$ & $\begin{array}{c}12040 \\
\pm 50 \mathrm{BP}\end{array}$ & $\begin{array}{c}12040- \\
11840 \\
B C\end{array}$ \\
\hline $\begin{array}{c}\text { Ziway } \\
2010\end{array}$ & Beta & 292527 & $\begin{array}{l}\text { DW1 } \\
\text { PS5 }\end{array}$ & Charbon & 10086 & $\overline{A M S}$ & $\begin{array}{c}29090 \\
\pm 160 \\
\mathrm{BP}\end{array}$ & $\begin{array}{c}29120 \\
\pm 160 \\
\text { BP }\end{array}$ & \\
\hline \multicolumn{10}{|c|}{ Échantillons géologiques } \\
\hline $\begin{array}{l}\text { Ziway } \\
2008\end{array}$ & Lyon & 6063 & $\begin{array}{c}\text { B2 } \\
\text { Bulbula } \\
\text { sequence }\end{array}$ & Coquillage & 6 & AMS & $\begin{array}{c}6000 \\
\pm 35 \mathrm{BP}\end{array}$ & & \\
\hline $\begin{array}{l}\text { Ziway } \\
2008\end{array}$ & Lyon & 6064 & $\begin{array}{c}\text { B2 } \\
\text { Bulbula } \\
\text { sequence }\end{array}$ & Coquillage & 7 & AMS & $\begin{array}{c}9970 \\
\pm 40 \mathrm{BP}\end{array}$ & & \\
\hline $\begin{array}{l}\text { Ziway } \\
2008\end{array}$ & Lyon & 6065 & $\begin{array}{c}\text { B2 } \\
\text { Bulbula } \\
\text { sequence }\end{array}$ & Coquillage & 8 & AMS & $\begin{array}{c}5985 \\
\pm 35 \text { ВР }\end{array}$ & & \\
\hline $\begin{array}{l}\text { Ziway } \\
2008\end{array}$ & Lyon & 6066 & $\begin{array}{c}\text { B2 } \\
\text { Tributary } \\
\text { sequence }\end{array}$ & Coquillage & 9 & AMS & $\begin{array}{c}6945 \\
\pm 35 \text { ВР }\end{array}$ & & \\
\hline $\begin{array}{l}\text { Ziway } \\
2008\end{array}$ & Lyon & 6067 & $\begin{array}{c}\text { B2 } \\
\text { Tributary } \\
\text { sequence }\end{array}$ & Coquillage & 10 & AMS & $\begin{array}{l}10430 \\
\pm 50 \mathrm{BP}\end{array}$ & & \\
\hline $\begin{array}{l}\text { Ziway } \\
2010\end{array}$ & Beta & 295900 & $\begin{array}{c}\text { B5 } \\
\text { G. Delta }\end{array}$ & Coquillage & 10074 & AMS & $\begin{array}{c}4420 \\
\pm 30 \mathrm{BP}\end{array}$ & $\begin{array}{c}4800 \\
\pm 30 \mathrm{BP}\end{array}$ & $\begin{array}{c}3650- \\
3520 \\
\text { BC }\end{array}$ \\
\hline $\begin{array}{l}\text { Ziway } \\
2010\end{array}$ & Beta & 295899 & Kurkura 2 & Coquillage & 10067 & AMS & $\begin{array}{c}41250 \\
\pm 420 \\
\text { BP }\end{array}$ & $\begin{array}{c}41640 \\
\pm 420 \\
\text { BP }\end{array}$ & \\
\hline
\end{tabular}




\section{Annexe 3 : Observation dans les abris d'Aga Dima (« Red Stone Hill 》)}

À quelque distance à l'ouest des rives de la Bulbula, s'élève une petite éminence rocheuse (cf. carte Fig. 1) ayant préalablement fait l'objet d'investigations archéologiques, il y a une trentaine d'années, qui révélèrent des traces d'occupation attribuables au LSA dans une grotte et un abri (Gallagher, 1977a). Cependant, compte-tenu de la faible ampleur des remplissages sédimentaires documentés alors, ces recherches n'avaient pas été en mesure de fournir des séquences archéologiques véritablement exploitables, et nous voulions nous rendre compte par nousmême de la nature exacte de ce contexte.

Le rocher d'Aga Dima est un petit relief de rhyolite, d'environ 500 mètres de long sur 250 mètres de large, dont le sommet domine de quelques 50 à 60 mètres la plaine environnante. Détaché d'environ $5 \mathrm{~km}$ vers l'est des hauteurs du Gademotta Ridge, il s'agit en fait de l'un des rares points hauts (environ 1720 mètres) existant au milieu de la plaine de la Bulbula. Ce rocher se décompose en trois petits sommets, entrecoupés de deux cols. Tantôt, des corniches escarpées en ferment l'accès; tantôt, des versants, quoique assez pentus, permettent assez aisément d'y accéder et de circuler sur l'ensemble de ce petit rocher.

Le trait de plus remarquable du rocher d'Aga Dima est la présence de nombreux abris naturels creusés dans les formations de rhyolite qui constituent sa principale ossature géologique. En effet, sur toute sa circonférence et à différentes hauteurs, se développent des abris, dont nous avons dénombré au moins une vingtaine. La plupart d'entre eux possèdent une très faible superficie : quelques mètres d'extension latérale pour 1 à 3 mètres de profondeur. Toutefois, il s'avère que certains d'entre eux sont relativement spacieux et, quoiqu'il en soit, la plupart ont manifestement été fréquentés au cours du LSA : sur les versants situés en contrebas de la majorité de ces abris comme sur leurs sols, lorsque ces derniers possèdent une couverture sédimentaire, une abondante industrie lithique peut être observée. Une partie des vestiges, en particulier ceux qui sont présents au bas des pentes, peuvent être attribués à une période très récente voir sub-actuelle (débitage d'éclats, grattoirs épais sur éclats). D'autres composantes industrielles, dominées par des productions de lames et de lamelles, attestent toutefois une fréquentation plus ancienne (LSA sensu lato), sans que nous puissions être plus précis sur le sujet.

Ainsi, outre les traces d'installations en plein air au sommet de petites collines (Waso, Macho) ou bien au creux de la plaine qu'elles surplombent (Bulbula et Deka Wede), il existe dans cette région un troisième contexte topographique abondamment fréquenté par des groupes du LSA : celui des abris sous roche dont le rocher d'Aga Dima nous a permis de mesurer l'intérêt. Malheureusement, à l'instar des observations préalablement effectuées par Gallagher, et même si nous nous sommes seulement 
contentés de prospecter les lieux sans entreprendre de sondages, il est manifeste que la plupart de ces abris comportent une très faible épaisseur de remplissage et nous devons conclure à notre tour que bien peu d'entre eux sont susceptibles de détenir de séquences archéologiquement exploitables. II faut toutefois mentionner la présence d'une véritable grotte, comportant un réseau souterrain bien développé, dans laquelle il serait davantage intéressant d'intervenir. Cependant, le fait qu'il s'agisse d'une tanière nauséabonde - peuplée de porcs-épics mais aussi, vraisemblablement, d'hyènes - la rend si peu hospitalière que nous avons renoncé à y entreprendre une opération.

\section{Annexe 4 : Note complémentaire à propos des « stones circles 》 observés à Waso Hill et Macho Hill}

Lors des prospections conduites en 2007 sur les sites de Macho Hill et Waso Hill (Bon et al., 2006), nous avions observé la présence de «stones circles » dont nous avions alors évoqué, parmi d'autres hypothèses, qu'il puisse s'agir d'aménagements funéraires. Cette hypothèse a été confortée en 2008 de plusieurs façons.

À Macho Hill, depuis notre visite de l'année précédente et devant I'intérêt que nous avions dû susciter sans nous en rendre compte à l'égard de ces «stones circles », un habitant du village voisin a effectué un grand trou au milieu de l'un d'entre eux. II pensait y découvrir un trésor mais, au lieu de cela, il mit au jour des ossements humains, ce qui lui causa apparemment un grand trouble et stoppa net ses espoirs comme ses investigations. Cette histoire, qui nous a été racontée par un de ses voisins (goguenard, selon nos souvenirs) nous accompagnant sur les lieux en 2008, a pu être confirmée par l'observation d'une dent humaine dans les déblais de l'excavation du malheureux chercheur de trésors.

À Waso Hill, nous avons procédé en 2008 à un micro-sondage à l'intérieur de l'une de ces structures, lequel a montré que le cercle de pierres visible en surface à cet endroit délimite également une fosse. Nous avons toutefois décidé de ne pas pousser davantage notre exploration de cette structure et ne pouvons donc préciser ni la forme ni la profondeur de cette fosse: notre équipe ne comptant aucun anthropologue spécialisé dans l'archéologie funéraire, nous avons jugé plus prudent de ne pas conduire une telle opération. Nous espérons aussi de cette façon ne pas avoir excité la convoitise des villageois de Waso, comme ce fut le cas de ceux de Macho.

Quoi qu'il en soit, et même si nous ne sommes donc pas en mesure d'apporter davantage de précisions sur ces structures funéraires d'un type apparemment inédit dans la partie centrale du Rift éthiopien, mais qui évoquent en revanche des découvertes similaires effectuées au Kenya sur le site de Ng'amoritung'a (Soper et Lynch, 1977), il nous a semblé intéressant de signaler cette découverte. 


\section{Bibliographie}

Barham L. \& Mitchell P., 2008, The First Africans: African archaeology from the Earliest Toolmakers to Most Recent Foragers, Cambridge World Archaeology.

Behailu Habte, 2013, A Terminal Pleistocene Cultural Sequence from Ethiopia: lithic analysis of B1s1 site, Bulbula River (Eastern Shewa), Master thesis, Addis Ababa University.

Benvenuti M., Carnicelli S., Belluomini G., Dainelli N., Di Grazia S., Ferrari G. A., lasio C., Sagri M., Ventra D., Balemwald Atnafu, Seifu Kebede, 2002, The Ziway-Shala Lake Basin (Main Ethiopian Rift, Ethiopia): a Revision of Basin Evolution with Special Reference to the Late Quaternary, Journal of African Earth Sciences, 35 (2), 247-269.

Bon F., Assamerew Dessie, Mensan R. \& Fauvelle-Aymar F.-X., 2006, Mission de prospection en archéologie préhistorique (LSA) dans la région des lacs d'Éthiopie (Koka, Ziway, Langano, Abijata), Annales d'Éthiopie, 2006 (2008), XXII, 85-129.

Bon F. \& Fauvelle-Aymar F.-X., 2013, Prehistory of East Africa, in Bahn P. \& Renfrew C. (ed.), The Cambridge World Prehistory, New York, Cambridge University Press.

Brandt S.A., 1986, The Upper Pleistocene and Early Holocene Prehistory of the Horn of Africa, African Archaeological Review, 4, 41-82.

Carnicelli S., Benvenuti M., Ferrari G. \& Sagri M., 2009, Dynamics and driving factors of late Holocene gullying in the Main Ethiopian Rift (MER), Geomorphology, 103, 541-554.

Douze K., 2012, Le Early Middle Stone Age d'Éthiopie et les changements techno-économiques à la période de l'émergence des premiers Homo sapiens, Thèse de l'université de Bordeaux I.

Gallagher J.P., 1972, A Preliminary Report on Archaeological Research near Lake Zuai, Annales d'Éthiopie, IX, 13-18.

Gallagher J.P., 1974, The Preparation of Hides with Stone Tools in South Central Ethiopia, Journal of Ethiopian studies, 13, 177-182.

Gallagher J.P., 1977a, Ethnoarchaeological and prehistoric investigations in the Ethiopian Central Rift Valley, Ph.D thesis, Southern Methodist University.

Gallagher J.P., 1977b, Contemporary Stone Tools in Ethiopia : Implications for Archaeology, Journal of Field Archaeology, 4, 407-414.

Gasse F., Rognon R. \& Street F.A., 1980, Quaternary History of the Afar and Ethiopian Rift Lakes, in Williams M.A.J. \& Faure H. (ed.), The Sahara and the Nile, Rotterdam, A.A. Balkema, 361-400. 
Gasse F. \& Street F.A., 1978, Late Quaternary Lake-Level Fluctuations in Environments of the Northern Rift Valley and Afar Regions (Ethiopia and Djibouti), Palaeogeography, Palaeoclimatology, Palaeoecology, 24, 279325.

Gillespie R., Street-Perrott F.A., Switsur R., 1983, Post-glacial arid episodes in Ethiopia have implications for climate prediction, Nature, 306, 680683.

Grove A.T., Street F.A. \& Goudie A.S., 1975, Former lake levels and climatic change in the Rift Valley of Southern Ethiopia, The Geographical Journal, 141 (2), 177-194.

Gutherz X., 2008, Aux origines de la production dans la corne de l'Afrique : un champ d'étude à reconquérir, Bulletin Archéologique de Provence, suppl. 5, 127-151.

Humphreys G., 1978, A Preliminary Report of Some Late Stone Age Occurrences in the Lake Ziway Area of the Central Ethiopian Rift valley, Annales d'Éthiopie, XI, 45-53.

Laury R.L. \& Albritton C.C., 1975, Geology of Middle Stone Age Archaeological Sites in the Main Ethiopian Rift Valley, Geological Society of America Bulletin, 86 (7), 999-1011.

Lane P., 2004, The 'moving frontier' and the transition to food production in Kenya, Azania: Archaeological Research in Africa, 39 (1), 243-264.

Le Turdu C., Tiercelin J.-J., Gibert E., Travi Y., Lezzar K.-E., Richert J.-P., Massault M., Gasse F., Bonnefille R., Decobert M., Gensous B., Jeudy V., Tamrat E., Mohammed M.U., Martens K., Atnafu B., Chernet T., Williamson D., \& Taieb M., 1999, The Ziway-Shala Lake Basin System, Main Ethiopian Rift: Influence of Volcanism, Tectonics, and Climate Forcing on Basin Formation and Sedimentation, Palaeogeography, Palaeoclimatology, Palaeoecology, 150, 135-177.

Ménard C., Bon F., Asamerew Dessie, Bruxelles L., Douze K., FauvelleAymar F.-X., Khalidi L. \& Mensan R., soumis, Late Stone Age variability in the Main Ethiopian Rift: new data from the Bulbula River, Ziway-Shala basin, Quaternary International.

Phillipson L., 2000, Aksumite Lithic Industries, African Archaeological Review, 17 (2), 49-63.

Rasmussen S.O., Andersen K.K., Svensson A.M., Steffensen J.P., Vinther B.M., Clausen H.B., Siggaard-Andersen M.-L., Johnsen S.J., Larsen L.B., Dahl-Jensen D., Bigler M., Röthlisberger R., Fischer H., Goto-Azuma K., Hansson M.E., Ruth U., 2006, A new Greenland ice core chronology for the last glacial termination, Journal of Geophysical Research, 111 (D06102). 
Reimer P.J., Baillie M.G.L., Bard E., Bayliss A., Beck J.W., Blackwell P.G., Ramsey C.B., Buck C.E., Burr G.S., Edwards R.L., Friedrich M., Grootes P.M., Guilderson T.P., Hajdas I., Heaton T.J., Hogg A.G., Hughen K.A., Kaiser K.F., Kromer B., McCormac F.G., Manning S.W., Reimer R.W., Richards D.A., Southon J.R., Talamo S., Turney C.S.M., Plicht J. van der, Weyhenmeyer C.E., 2011, IntCal09 and Marine09 Radiocarbon Age Calibration Curves, 0-50,000 Years cal BP, Radiocarbon, 51, 1111-1150.

Sagri M., Bartolini C., Billi P., Ferrari G., Benvenuti M., Carnicelli S. \& Barbano F., 2008, Latest Pleistocene and Holocene river network evolution in the Ethiopian Lakes region, Geomorphology, 94, 79-97.

Sahle Y., Morgan L. E., Braun D. R., Balemwal Atnafu \& Hutchings W. K., sous presse, Chronological and behavioral contexts of the earliest Middle Stone Age in the Gademotta Formation, Main Ethiopian Rift, Quaternary <http://dx.doi.org/10.1016/j.quaint.2013.03.010> International.

Soper R. \& Lynch M., 1977, The Stone-Circle Graves at Ng'amoritung'a, Southern Turkana District, Kenya, Azania, XII, 193-211.

Street F.A., 1979, Late Quaternary lakes in the Ziway-Shala Basin, Southern Ethiopia", PhD thesis, University of Cambridge.

Sutton J.E.G., 1977, The African aqualithic, Antiquity, LI, 25-34.

Umer, M., Legesse, D., Gasse, F., Bonnefille, R., Lamb, H., Leng, M.J. \& Lamb, A.L., 2004, Late Quaternary climate changes in the Horn of Africa, in Battarbee R.W., Gasse, F., Stickley, C.E. (ed.), Past Climate Variability through Europe and Africa, Developments in Paleoenvironmental Research, 6, Springer, 159-180.

Wendorf F. \& Schild R., 1974, A Middle Stone Age Sequence from the central Rift Valley, Ethiopia, Polska Akademia Nauk, Wroclaw, Ossolineum. 


\section{Résumé / Abstract}

Bon F. et al., 2013, Archéologie préhistorique de la partie centrale du Main Ethiopian Rift (bassin lacustre de Ziway-Shala) : contribution à l'établissement de la séquence Late Stone Age d'Afrique orientale, Annales d'Éthiopie, 28, 261-297.

Soutenue par le CFEE, le ARCCH, le MAEE, l'INRAP et le laboratoire TRACES, la mission "Late Stone Age sequence in Ethiopia » est dédiée à la réunion de nouvelles données sur la préhistoire récente de la Corne de l'Afrique. Afin de répondre à cet objectif, plusieurs campagnes de terrain se sont déroulées depuis 2007 dans la région des lacs Ziway, Abijata et Langano, situés au coeur de la vallée du Rift, profitant des différents contextes d'enregistrement géologique que recèle cette région et, en particulier, les contextes fluviolacustres de la plaine de la Bulbula. En effet, cette zone offre un terrain très propice à l'étude de la relation de l'Homme avec son milieu naturel et ses variations, parfois brutales, qu'il s'agisse des fluctuations hydrométriques comme des phénomènes de type " catastrophiques » imputables à l'activité volcanique. Grâce au programme pluridisciplinaire que nous avons pu développer dans une région possédant donc une haute résolution d'enregistrement sédimentaire et archéologique, cette mission est parvenue à compléter significativement son cadre géomorphologique et géologique, achevant l'établissement d'une séquence stratigraphique au sein de laquelle ont été découverts plusieurs nouveaux sites archéologiques, dont certains livrent des informations proprement inédites sur la Préhistoire de l'Éthiopie.

Nous retiendrons en particulier les documents fournis par plusieurs sites datés des environs de 30000 BP ainsi que sur la charnière Pléistocène/Holocène (entre 12 et 9000 cal BC environ). Cet enregistrement archéologique est par essence discontinu, puisqu'il témoigne de phases d'occupation rythmées par les grands cycles climatiques de transgression et de régression lacustre ou, corrélativement, de plus ou moins forte aridité ; la présence humaine fut également dépendante des épisodes volcaniques qui ont pu momentanément bouleverser les conditions de vie dans cette zone. Toutefois, chacune des phases d'occupation décelées apportent des éclairages nouveaux à la connaissance de la Préhistoire éthiopienne, qu'il s'agisse de contribuer à l'identification d'une phase finale du MSA lors de l'OIS3 comme de documenter la diversité des expressions techniques et économiques du LSA (autour notamment du rôle respectif de la chasse et de la pêche) à la charnière du Pléistocène et de l'Holocène. Parallèlement à cela, un programme étroitement complémentaire concernant la gîtologie des sources d'obsidienne environnantes a été développé.

Mots-clefs : Éthiopie, Bassin lacustre de Ziway-Shala, Bulbula River, Final Middle Stone Age, Late Stone Age, archéologie, géoarchéologie, pétroarchéologie

Prehistory of the Central Main Ethiopian Rift (Ziway-Shala basin): Establishing the Late Stone Age sequence in Eastern Africa - Supported by the CFEE, ARCCH, MAEE, INRAP and the laboratory TRACES, this project, "Late Stone Age sequence in Ethiopia", is devoted to the collection of new data on the late prehistory of the Horn of Africa. To answer to this objective, several fieldwork seasons have been carried out since 2007 in the area of lakes Ziway, Langano and Abijata, located in the central part of the Main Ethiopian Rift Valley. Over the course of this project, we have intensively explored different geological contexts, and in particular the fluvio-lacustrine plain of the Bulbula river and the impact of volcanic eruption on 
the landscape and on human acquisition strategies. This area provides an ideal terrain for the study of prehistoric human/environment relationships in the context of a shifting natural environment (lake fluctuations / volcanic activity). This collaborative program, developed in a region with a uniquely rich and well preserved sedimentary and archaeological record, has supplemented new data establishing the geomorphological and geological setting, with a stratigraphic sequence in which several new archaeological sites have been discovered and studied.

Most notable are several sites dating to around $30,000 \mathrm{BP}$, a period previously poorly documented in Ethiopian Prehistory, and several sites dating to the transition between the Pleistocene and Holocene periods (between 12,000 and 9,000 cal BC). The archaeological sequence we have identified in the Bulbula plain is discontinuous because it is linked to lakelevel fluctuations but also to arid and volcanic events. Nevertheless, the different windows of preservation identified shed new light on a variety of issues regarding the late Prehistory of this area. It concerns particularly a final stage of the MSA (contemporary OIS3) and diverse expressions of the LSA. The signature of this diversity is both technical and economical, around the role of hunting and fishing practices. This interdisciplinary project combines geomorphological and archaeological perspectives with detailed work on raw material sampling and analysis.

Keywords: Ethiopia, Ziway-Shala basin, Bulbula river, Final Middle Stone Age, Late Stone Age, archaeology, geoarchaeology, petroarchaeology 